\title{
Total Prompt Energy Release in the Neutron-Induced Fission of ${ }^{235} \mathbf{U},{ }^{238} \mathbf{U}$, and ${ }^{239} \mathbf{P u}$
}

\author{
D. G. Madland \\ Theoretical Division, Los Alamos National Laboratory, Los Alamos, New Mexico \\ 87545
}

\begin{abstract}
This study addresses, for the first time, the total prompt energy release and its components for the fission of ${ }^{235} \mathrm{U},{ }^{238} \mathrm{U}$, and ${ }^{239} \mathrm{Pu}$ as a function of the kinetic energy of the neutron inducing the fission. The components are extracted from experimental measurements, where they exist, together with model-dependent calculation, interpolation, and extrapolation. While the components display clear dependencies upon the incident neutron energy, their sums display only weak, yet definite, energy dependencies. Also addressed is the total prompt energy deposition in fission for the same three systems. Results are presented in equation form. New measurements are recommended as a consequence of this study.
\end{abstract}

Key words: Energy release and energy deposition in neutron-induced fission, experiment and Los Alamos model, ${ }^{235} \mathrm{U},{ }^{238} \mathrm{U},{ }^{239} \mathrm{Pu}$

PACS: 24.75.+i, 25.85.Ec, 25.85.Ca, 27.90.+b

\section{Introduction}

This study is a consequence of open questions on the magnitudes of the total prompt energy release in fission, the total prompt energy deposition in fission, the components of these quantities, and their dependencies upon the kinetic energy of the neutron inducing the fission. Our results are given in Eqs. (31 - 33) and Fig. 17 for the total prompt energy release in fission, and in Eqs. (46 - 48) and Fig. 23 for the total prompt energy deposition in fission. Recommended new experimental measurements coming from this study are given in Sec. 6. It should be noted that the study relies primarily upon existing published experimental measurements and secondarily upon nuclear theory and nuclear models. Therefore, new and higher quality measurements would 
improve the results presented here and, furthermore, would lead to a more complete understanding of post scission fission physics.

\section{Energy Conservation}

The energy release in fission is obtained from energy conservation. If one considers the binary fission of an actinide nucleus of mass number $A-1$ induced by a neutron of mass $m_{n}$, kinetic energy $E_{n}$, and binding energy $B_{n}$ in the

compound nucleus $A$ formed when the neutron is absorbed, then energy conservation gives

$$
\begin{aligned}
E_{n}+m_{n}+M(Z, A-1) & =T+M^{*}(Z, A) \\
& =T_{L}\left(Z_{L}, A_{L}\right)+M_{L}^{*}\left(Z_{L}, A_{L}\right) \\
& +T_{H}\left(Z_{H}, A_{H}\right)+M_{H}^{*}\left(Z_{H}, A_{H}\right)
\end{aligned}
$$

where the left side specifies the initial conditions prior to fission, the right side specifies the excited compound nucleus that is about to fission, and the second right side specifies the excited fission fragments just after binary fission has occurred. The notation here is that $T$ is a compound nucleus or fragment kinetic energy, $M$ and $m$ are stable masses, $M^{*}$ are masses of excited nuclei, all in units of $\mathrm{MeV}\left(c^{2}\right.$ has been suppressed), and $L$ and $H$ refer to the light and heavy fragments occurring in the binary fission.

The neutron binding energy $B_{n}$ is obtained from the Q-value for neutron capture,

$$
m_{n}+M(Z, A-1)=M(Z, A)+B_{n}
$$

which, when inserted into Eq. (1), yields

$$
\begin{aligned}
E_{n}+B_{n}+M(Z, A) & =T_{L}\left(Z_{L}, A_{L}\right)+T_{H}\left(Z_{H}, A_{H}\right)+M_{L}\left(Z_{L}, A_{L}\right) \\
& +M_{H}\left(Z_{H}, A_{H}\right)+E_{L}^{*}\left(Z_{L}, A_{L}\right)+E_{H}^{*}\left(Z_{H}, A_{H}\right)
\end{aligned}
$$

where we have written an excited fragment mass as the ground-state mass plus the excitation energy, namely, $M^{*}=M+E^{*}$. This excitation energy will be dissipated by the emission of prompt neutrons and prompt gamma rays.

The total energy release $E_{r}$ in binary fission is defined as the ground-state mass of the compound nucleus undergoing fission minus the ground-state masses of 
the two binary fission fragments, namely,

$$
E_{r}=M(Z, A)-M_{L}\left(Z_{L}, A_{L}\right)-M_{H}\left(Z_{H}, A_{H}\right)
$$

where (again) the masses are expressed in units of $\mathrm{MeV}$ [1]. This equation also defines the total energy release in each stage of multiple-chance fission except for the average kinetic and binding energies of the neutron(s) emitted prior to fission in each stage, which must be taken into account. Inserting Eq. (4) into Eq. (3) yields a second expression for the energy release in fission:

$$
E_{r}=T_{L}\left(Z_{L}, A_{L}\right)+T_{H}\left(Z_{H}, A_{H}\right)+E_{L}^{*}\left(Z_{L}, A_{L}\right)+E_{H}^{*}\left(Z_{H}, A_{H}\right)-\left(E_{n}+B_{n}\right)
$$

In these equations, conservation of charge ensures that $Z=Z_{L}+Z_{H}$, and conservation of baryon number ensures that $A=A_{L}+A_{H}$. Note that in Eq. (5) the kinetic and binding energy of the neutron inducing fission explicitly appear, with a minus sign, but they also implicitly appear in the fragment excitation energies $E_{L}^{*}$ and $E_{H}^{*}$. And for spontaneous fission one replaces $\left(E_{n}+\right.$ $B_{n}$ ) with 0 , while for photofission the replacement is $E_{\gamma}$.

If we now sum the fragment kinetic energies and fragment excitation energies, Eq. (5) becomes

$$
E_{r}=T_{f}^{t o t}+E_{t o t}^{*}-\left(E_{n}+B_{n}\right)
$$

with

$$
\begin{aligned}
& T_{f}^{\text {tot }}=T_{L}\left(Z_{L}, A_{L}\right)+T_{H}\left(Z_{H}, A_{H}\right) \\
& E_{\text {tot }}^{*}=E_{L}^{*}\left(Z_{L}, A_{L}\right)+E_{H}^{*}\left(Z_{H}, A_{H}\right) .
\end{aligned}
$$

The above equations are for the specific binary fission $\left(Z_{L}, A_{L}\right)+\left(Z_{H}, A_{H}\right)$.

However, a large number of binary mass splits are energetically allowed and they have been observed throughout the pre-actinide, actinide, and transactinide regions [2]. In the vicinity of the uranium and plutonium isotopes the fission-fragment mass range is (approximately) $70 \leq A_{f} \leq 170$ and for each $A_{f}$ there are 4 to 5 contributing isobars leading to between 200 and 250 different possible mass splits in binary fission ( $f$ stands for fragment). This means that the total energy release $E_{r}$, the total fission-fragment kinetic energy $T_{f}^{t o t}$, and the total fission-fragment excitation energy $E_{\text {tot }}^{*}$, in Eqs. (4-8), 
must be replaced by their average values as determined by weighting with the independent fission-fragment yields $Y_{f}$, where

$$
Y_{f}\left(Z_{L}, A_{L}\right)=Y_{f}\left(Z-Z_{L}, A-A_{L}\right)=Y_{f}\left(Z_{H}, A_{H}\right)
$$

leading to

$$
\begin{aligned}
\left\langle E_{r}\right\rangle & =M(Z, A)-\frac{\sum\left[Y_{f}\right]\left[M_{L}\left(Z_{L}, A_{L}\right)+M_{H}\left(Z_{H}, A_{H}\right)\right]}{\sum Y_{f}} \\
& =\left\langle T_{f}^{\text {tot }}\right\rangle+\left\langle E_{\text {tot }}^{*}\right\rangle-\left(E_{n}+B_{n}\right)
\end{aligned}
$$

with

$$
\begin{aligned}
\left\langle T_{f}^{t o t}\right\rangle & =\frac{\sum\left[Y_{f}\right]\left[T_{L}\left(Z_{L}, A_{L}\right)+T_{H}\left(Z_{H}, A_{H}\right)\right]}{\sum Y_{f}} \\
\left\langle E_{\text {tot }}^{*}\right\rangle & =\frac{\sum\left[Y_{f}\right]\left[E_{L}^{*}\left(Z_{L}, A_{L}\right)+E_{H}^{*}\left(Z_{H}, A_{H}\right)\right]}{\sum Y_{f}}
\end{aligned}
$$

and the sums are understood to be over either the light $\{L\}$ or the heavy $\{H\}$ fission-fragment yields. Thus, Eqs. (9-13) replace Eqs. (4-8) for the total energy release in binary fission. Note, again, that in the case of spontaneous fission $E_{n}$ and $B_{n}$ in Eq. (11) are set to zero, while in the case of photofission they are replaced with $E_{\gamma}$.

Extraction of the average total prompt energy release in fission from Eqs. (913) requires consideration of the time dependence of the fission process and the introduction of definitions related to that time dependence. A schematic of neutron-induced binary fission is shown in Fig. 1. The terms appearing in the figure, as well as others, are defined as follows:

scission point: The time at which the fission fragments are unalterably determined [3]. Loosely, the time at which the neck snaps between the nascent fission fragments.

fission fragment: Nuclear species existing at the scission point and just beyond, but prior to the emission of prompt neutrons and prompt gamma rays.

fission fragment acceleration time: $\sim 10^{-20}$ [sec] due to Coulomb repulsion. prompt neutron emission time: In the range $\sim 10^{-18}$ to $\sim 10^{-13}$ [sec] based upon measurement of compound nucleus lifetimes and calculation. prompt gamma emission time: In the range $\sim 10^{-14}$ to $\sim 10^{-7}$ [sec] based upon time-of-flight measurements and calculation. See Ref. [4]. 


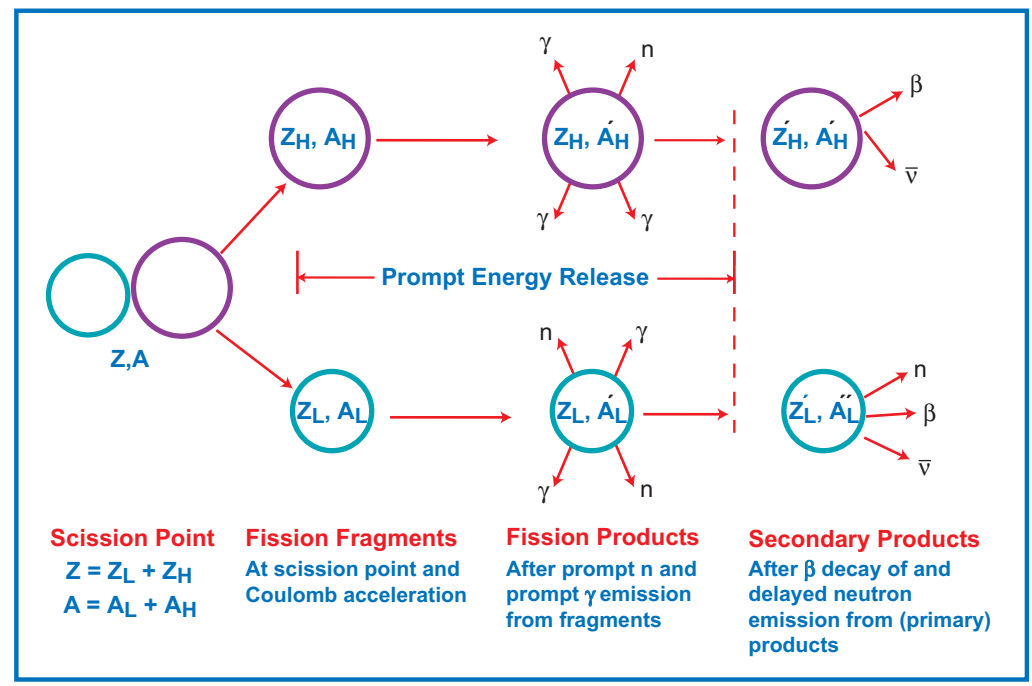

Fig. 1. Schematic of post scission in neutron-induced binary fission of target nucleus $(Z, A-1)$.

prompt energy release time: In the range $\sim 10^{-20}$ to $\sim 10^{-7}$ [sec]. See Ref. [4]. fission product (or primary fission product): Nuclear species existing following prompt neutron emission and prompt gamma emission from a fragment, but before any $\beta$ decay has occurred.

secondary fission product: Nuclear species existing following at least one $\beta$ decay of a primary fission product. The shortest known fission-product $\beta$ decay half-life is 0.032 [sec]. Therefore, secondary fission products, $\beta$-decay energy spectra, antineutrino energy spectra, and subsequent delayed neutron energy spectra play no role in the total prompt fission energy release.

Now the independent fission-fragment yields $Y_{f}\left(Z_{f}, A_{f}\right)$ required in the solution of Eqs. (9-13) can only be obtained by construction from the measured independent fission-product yields $Y_{p}\left(Z_{p}, A_{p}\right)$ (where $p$ stands for product), the measured average prompt neutron multiplicity as a function of fission-fragment mass $\bar{\nu}_{p}\left(A_{f}\right)$ (where $p$ stands for prompt), and a Gaussian or Gaussian-like model assumption for the $Z_{f}$ dependence of $Y_{f}$ for fixed $A_{f}$. Note that $Z_{p}=Z_{f}$, but that $A_{p} \leq A_{f}$ due to the prompt neutron emission from the fragment. Furthermore, the independent fission-product yields $Y_{p}\left(Z_{p}, A_{p}\right)$ have been extensively measured, and tabulated, only for spontaneous fission and neutroninduced fission at two well-defined energies, thermal and $14 \mathrm{MeV}$ (these are the well-known double-humped mass yield $\left(A_{p}\right)$ distributions together with Gaussian charge yield $\left(Z_{p}\right)$ distributions) [6]. Thus, solution of Eqs. (9-13) by use of constructed independent fission-fragment yields $Y_{f}\left(Z_{f}, A_{f}\right)$ is not currently tractable except for incident thermal neutrons and $14-\mathrm{MeV}$ neutrons. Therefore, we turn to direct use of measured and/or calculated values of the 
terms appearing in Eq. (11). Before proceeding we note the following:

multiple-chance fission: Insofar as measured quantities are used in the evaluation of Eq. (11) the effects of multiple-chance fission are automatically taken into account. However, as will be seen, some calculated quantities are yet needed and these require multiple-chance fission treatment [see Eqs. (24) and (42)]. Appendix A contains the multiple-chance fission equations to be used in the calculation of the average total prompt fission energy release and energy deposition, $\left\langle E_{r}\right\rangle$ and $\left\langle E_{d}\right\rangle$ respectively, when none of the measured components of these quantities are available.

ternary fission: For the incident neutron energy range $0 \leq E_{n} \leq 15 \mathrm{MeV}$, approximately 1 in 500 fissions is ternary [7]. Therefore, ternary fission has been ignored in the preceeding equations. However, insofar as ternary fission events have affected the measurements to be used below, their influence is present. Here, ternary means light charged particle accompanied fission.

scission neutrons: The question of neutron emission at the scission point remains an open one, with experimental results ranging from $0 \%$ to $10 \%$ of the total average prompt neutron multiplicity $\bar{\nu}_{p}[7]$. Measurements of this quantity, to be used below, include the scission neutrons if they exist.

isomeric states: The de-excitation of fission fragments to (long-lived) isomeric states has been ignored in the preceeding equations and time definitions. However, their effects are included insofar as they affect the measurements to be used below.

\section{Components of the Average Total Prompt Fission Energy Re- lease}

The components of the average total prompt fission energy release $\left\langle E_{r}\right\rangle$ to be evaluated for the solution of Eq. (11) are the average total fission-fragment kinetic energy $\left\langle T_{f}^{\text {tot }}\right\rangle$ and the average total fission-fragment excitation energy $\left\langle E_{\text {tot }}^{*}\right\rangle$ whereas $E_{n}$ and $B_{n}$ are known. The largest component is the average total fission-fragment kinetic energy $\left\langle T_{f}^{t o t}\right\rangle$ which becomes, after prompt neutron emission times ranging from $10^{-18}$ to $10^{-13}$ [sec], the average total fission-product kinetic energy $\left\langle T_{p}^{t o t}\right\rangle$ which is the measured quantity. More often than not, the experimentalists have converted the measured product kinetic energies back to fragment kinetic energies because they are more relevant to the development of fission theory. These quantities are related by the kinetic energies of the prompt neutrons emitted from the moving fragments as they become moving products. As a function of the kinetic energy $E_{n}$ of the 
neutron inducing fission, one obtains

$$
\left\langle T_{p}^{t o t}\left(E_{n}\right)\right\rangle=\left\langle T_{f}^{t o t}\left(E_{n}\right)\right\rangle\left[1-\frac{\bar{\nu}_{p}\left(E_{n}\right)}{2 A}\left(\frac{\left\langle A_{H}\right\rangle}{\left\langle A_{L}\right\rangle}+\frac{\left\langle A_{L}\right\rangle}{\left\langle A_{H}\right\rangle}\right)\right]
$$

Equation (14) yields about a $2 \%$ kinetic energy correction due to prompt neutron emission from fully accelerated fragments coming from $14-\mathrm{MeV}$ neutroninduced fission. The approximations used in its derivation are:

a) $m_{n} / M(Z, A)=1 / A$

b) $\bar{\nu}_{p}(L)=\bar{\nu}_{p}(H)=\bar{\nu}_{p} / 2$ where $L$ and $H$ refer to the average light and average heavy fragments

c) $T_{f} / A=T_{f 1} /(A-1)=T_{f 2} /(A-2)=\ldots$ where $T_{f}$ is the initial fragment kinetic energy of the initial fragment $A$, and $T_{f i}$ are fragment kinetic energies following the $i$ th neutron evaporation from the moving fragment.

These approximations are sufficiently accurate to quantify a correction of order $2 \%$.

The average total fission-fragment excitation energy $\left\langle E_{t o t}^{*}\right\rangle$ is dissipated by two separate mechanisms: prompt neutron emission and prompt gamma emission, where prompt time has already been specified for each mechanism. Thus,

$$
\left\langle E_{\text {tot }}^{*}\right\rangle=\left\langle E x_{n}^{\text {tot }}\right\rangle+\left\langle E_{\gamma}^{\text {tot }}\right\rangle
$$

where the average fission-fragment excitation energy leading to prompt neutron emission is given by [5]

$$
\left\langle E x_{n}^{t o t}\right\rangle=\bar{\nu}_{p}\left[\left\langle S_{n}\right\rangle+\langle\varepsilon\rangle\right]
$$

with $\left\langle S_{n}\right\rangle$ the average fission-fragment neutron separation energy and $\langle\varepsilon\rangle$ the average center-of-mass energy of the emitted neutrons, and the average fissionfragment excitation energy leading to prompt gamma emission is given by $\left\langle E_{\gamma}^{\text {tot }}\right\rangle$. Equation (11) now becomes

$$
\begin{aligned}
\left\langle E_{r}\right\rangle & =\left\langle T_{f}^{t o t}\right\rangle+\left\langle E x_{n}^{t o t}\right\rangle+\left\langle E_{\gamma}^{t o t}\right\rangle-\left(E_{n}+B_{n}\right) \\
& =\left\langle T_{f}^{t o t}\right\rangle+\bar{\nu}_{p}\left[\left\langle S_{n}\right\rangle+\langle\varepsilon\rangle\right]+\left\langle E_{\gamma}^{t o t}\right\rangle-\left(E_{n}+B_{n}\right)
\end{aligned}
$$

Note that all averaged quantities appearing in Eqs. (15-17) depend upon the incident neutron energy $E_{n}$ which has been suppressed for brevity. We use Eq. 
(17) for the average total prompt energy release in neutron-induced fission for the remainder of this paper.

The existing experimental database for the neutron-induced fission of ${ }^{235} \mathrm{U}$, ${ }^{238} \mathrm{U}$, and ${ }^{239} \mathrm{Pu}$, together with model-dependent interpolation, extrapolation, and calculation, allow a determination of $\left\langle E_{r}\right\rangle$ over the incident neutron energy range of $0 \leq E_{n} \leq 15 \mathrm{MeV}$. However, as will be seen below, the experimental database is astonishingly incomplete. Where experiment does exist, we have performed linear or quadratic least-squares fits in $E_{n}$ to the data and present the resulting parameters and their standard deviations in the following. Standard (theoretical) deviations in the Los Alamos model, as used in the following, are not quantitatively addressed herein.

\subsection{Average Total Fission Fragment and Fission Product Kinetic Energy}

The experimental data that we use for the $\mathrm{n}+{ }^{235} \mathrm{U}$ system are those of Meadows and Budtz-Jorgensen (1982) [8], Straede et al. (1987) [9], and Müller et al. (1984, two data points only) [10]. The published fission-fragment (pre prompt neutron emission) total kinetic energies are shown in Fig. 2 and the corresponding fission-product (post prompt neutron emission) total kinetic energies, obtained with Eq. (14), are shown in Fig. 3. Linear fits to these data are also shown in the figures.

For the $\mathbf{n}+{ }^{235} \mathbf{U}$ system:

$$
\begin{aligned}
& \left\langle T_{f}^{\text {tot }}\right\rangle=(170.93 \pm 0.07)-(0.1544 \pm 0.02) E_{n}(\mathrm{MeV}) \\
& \left\langle T_{p}^{\text {tot }}\right\rangle=(169.13 \pm 0.07)-(0.2660 \pm 0.02) E_{n}(\mathrm{MeV})
\end{aligned}
$$

The data appear to have structure (near the second-chance fission threshold, for example), but their scatter and uncertainties preclude anything other than a linear fit. The steeper negative slope for the total fission-product kinetic energy is due to the energy dependence of $\bar{\nu}_{p}$ in Eq. (14). Strictly, Eqs. (18) and (19) should not be used above an incident neutron energy of about $9 \mathrm{MeV}$.

The experimental data that we use for the $\mathrm{n}+{ }^{238} \mathrm{U}$ system are those of Zöller (1995) [11] shown in Fig. 4 and Fig. 5 for incident neutron energies up to $30 \mathrm{MeV}$. Here, the data give clear and convincing evidence for the presence of structure near the second- and third-chance fission thresholds. For our present purposes, however, we represent these data with quadratic fits for both the total fission-fragment and total fission-product kinetic energies because the corresponding experimental data for ${ }^{235} \mathrm{U}$ and ${ }^{239} \mathrm{Pu}$ are much lower in quality and over more limited energy ranges. We note that the recent 
experimental data of Vivès et al. (2000) [12] for this system, over an incident neutron energy range of $1.2-5.8 \mathrm{MeV}$, are in substantial agreement with the corresponding data of Zöller (Fig. 4). The maximum discrepancy between the two measurements is $\sim 0.7 \%$ at about $1.5 \mathrm{MeV}$. 


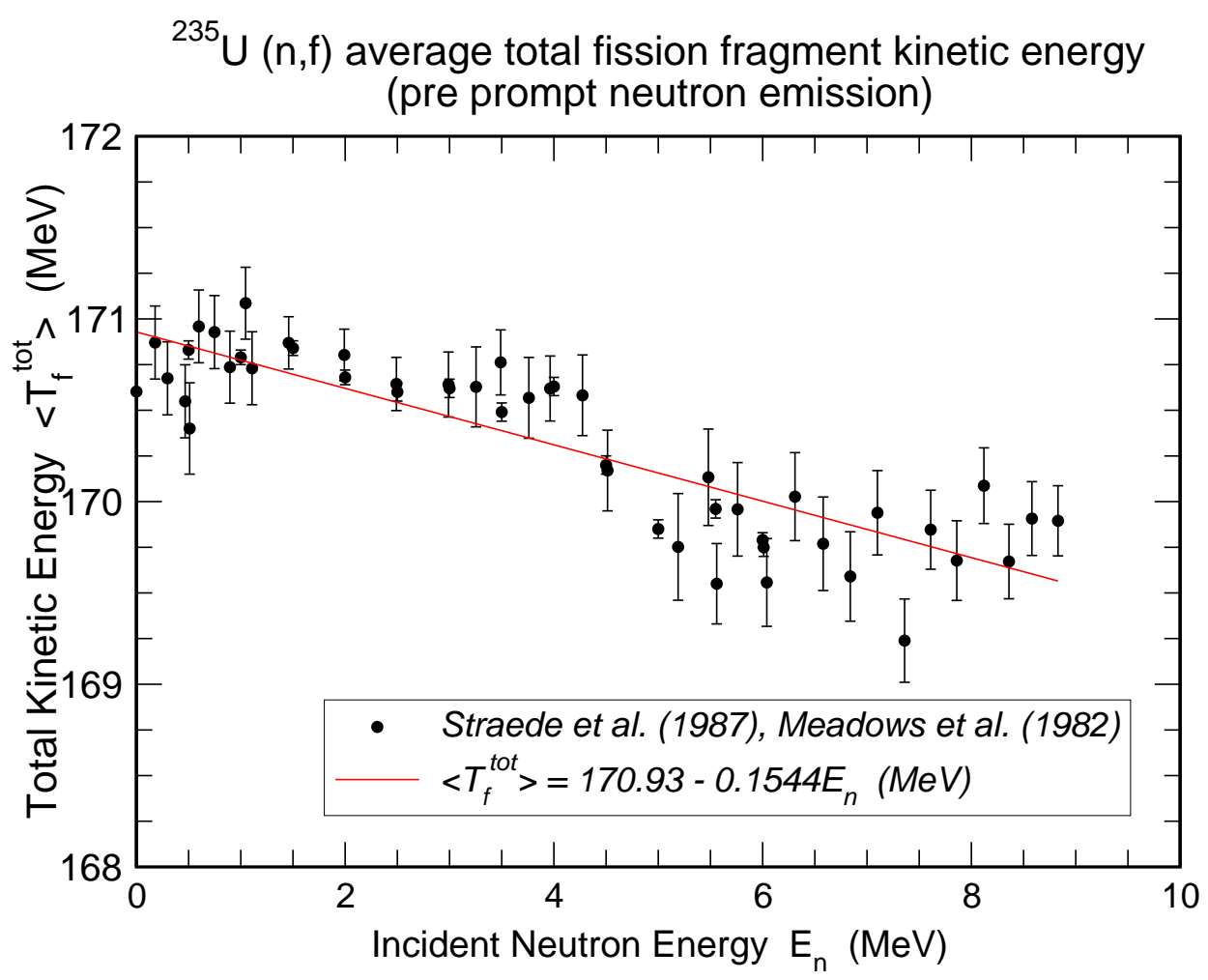

Fig. 2. Average total fission-fragment kinetic energy for the $n\left(E_{n}\right)+{ }^{235} U$ system.

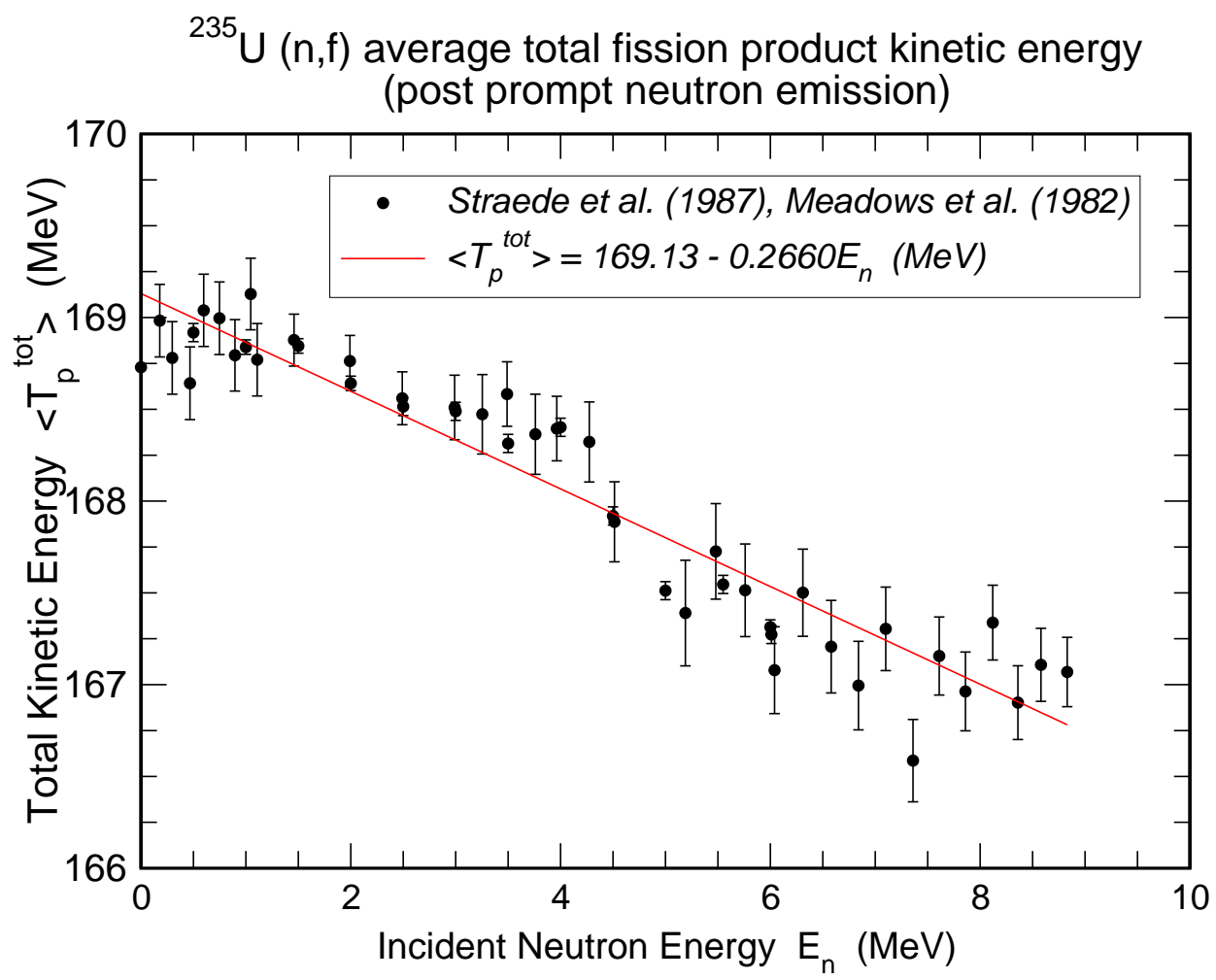

Fig. 3. Average total fission-product kinetic energy for the $n\left(E_{n}\right)+{ }^{235} U$ system. 


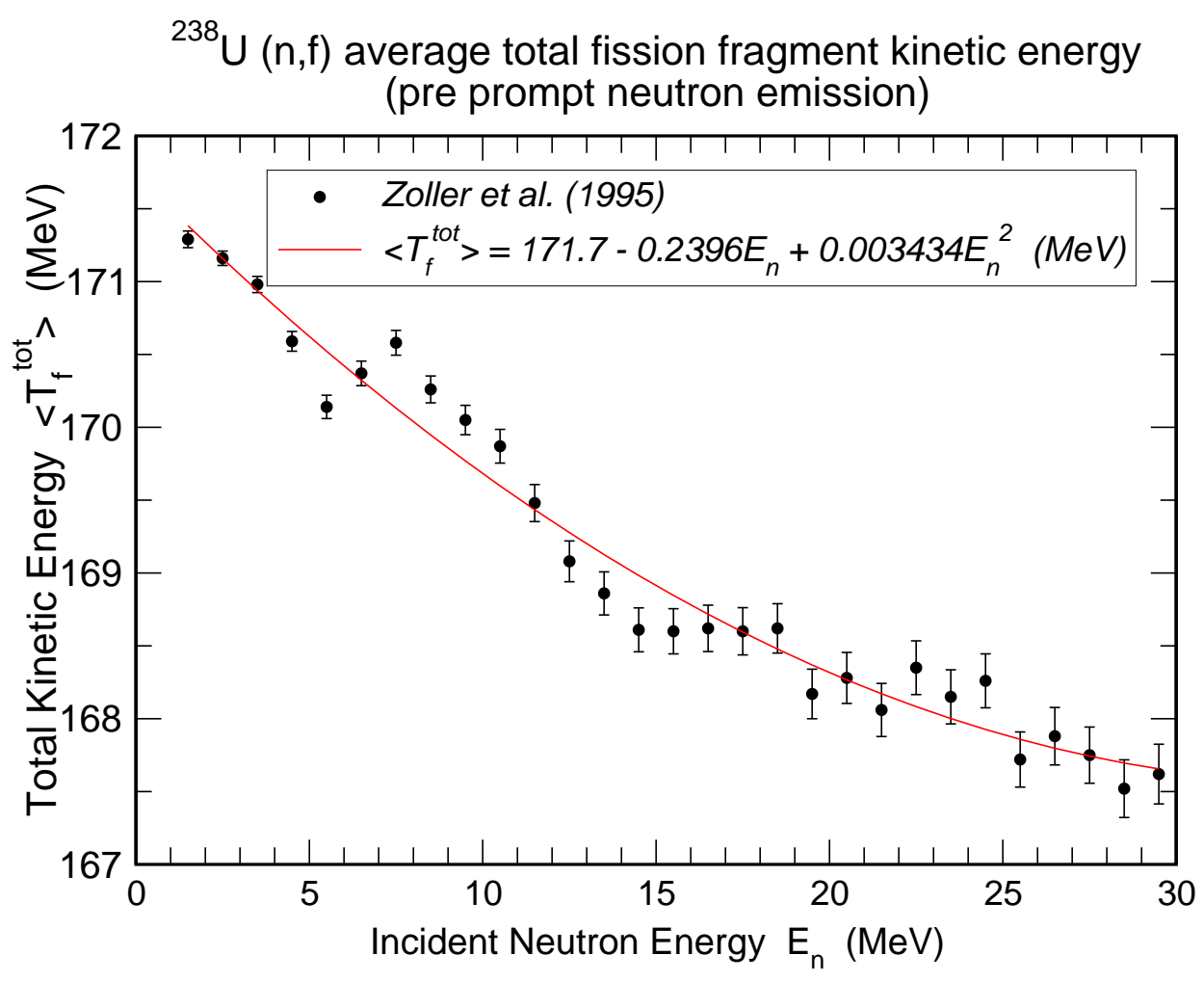

Fig. 4. Average total fission-fragment kinetic energy for the $n\left(E_{n}\right)+{ }^{238} U$ system.

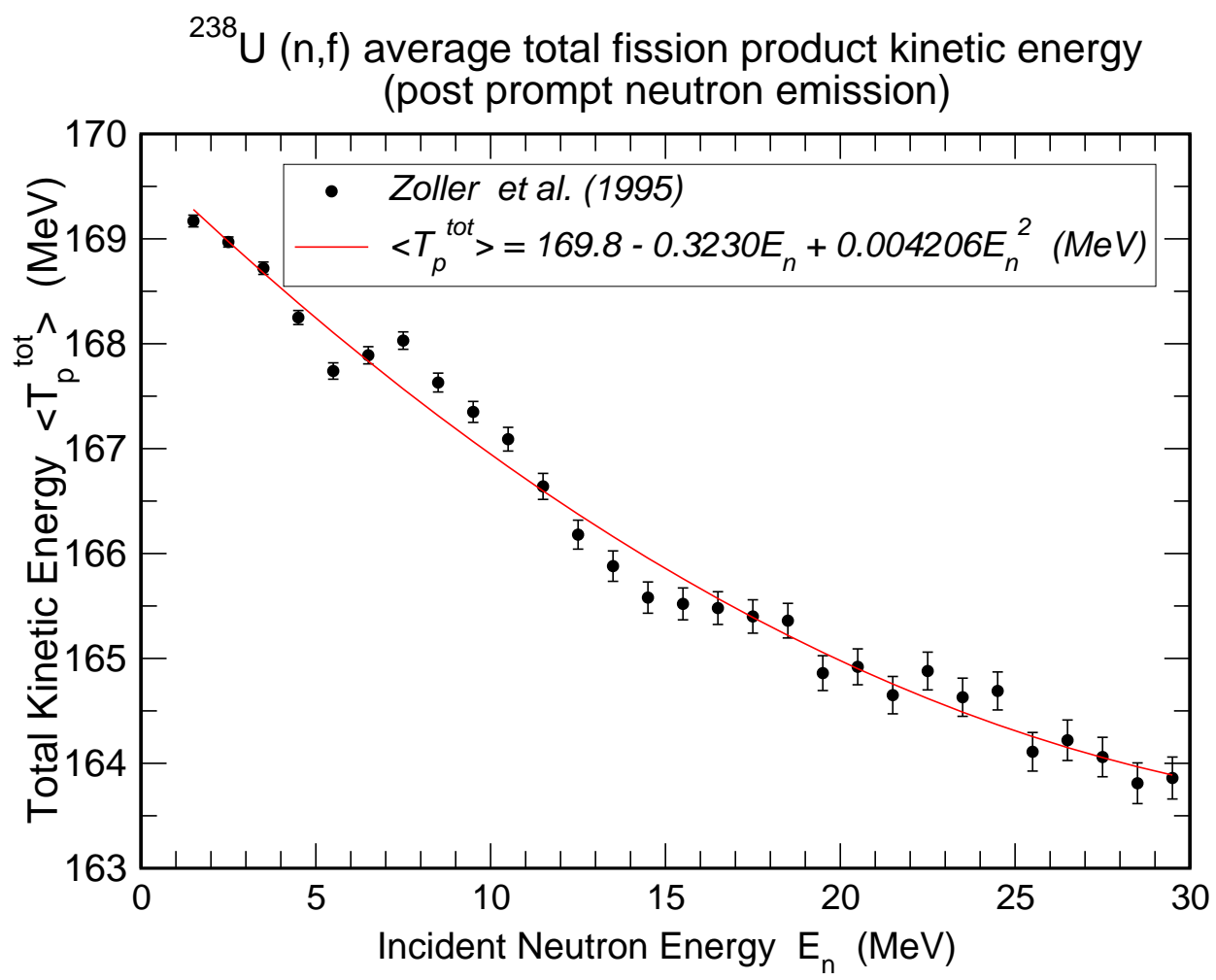

Fig. 5. Average total fission-product kinetic energy for the $n\left(E_{n}\right)+{ }^{238} U$ system. 


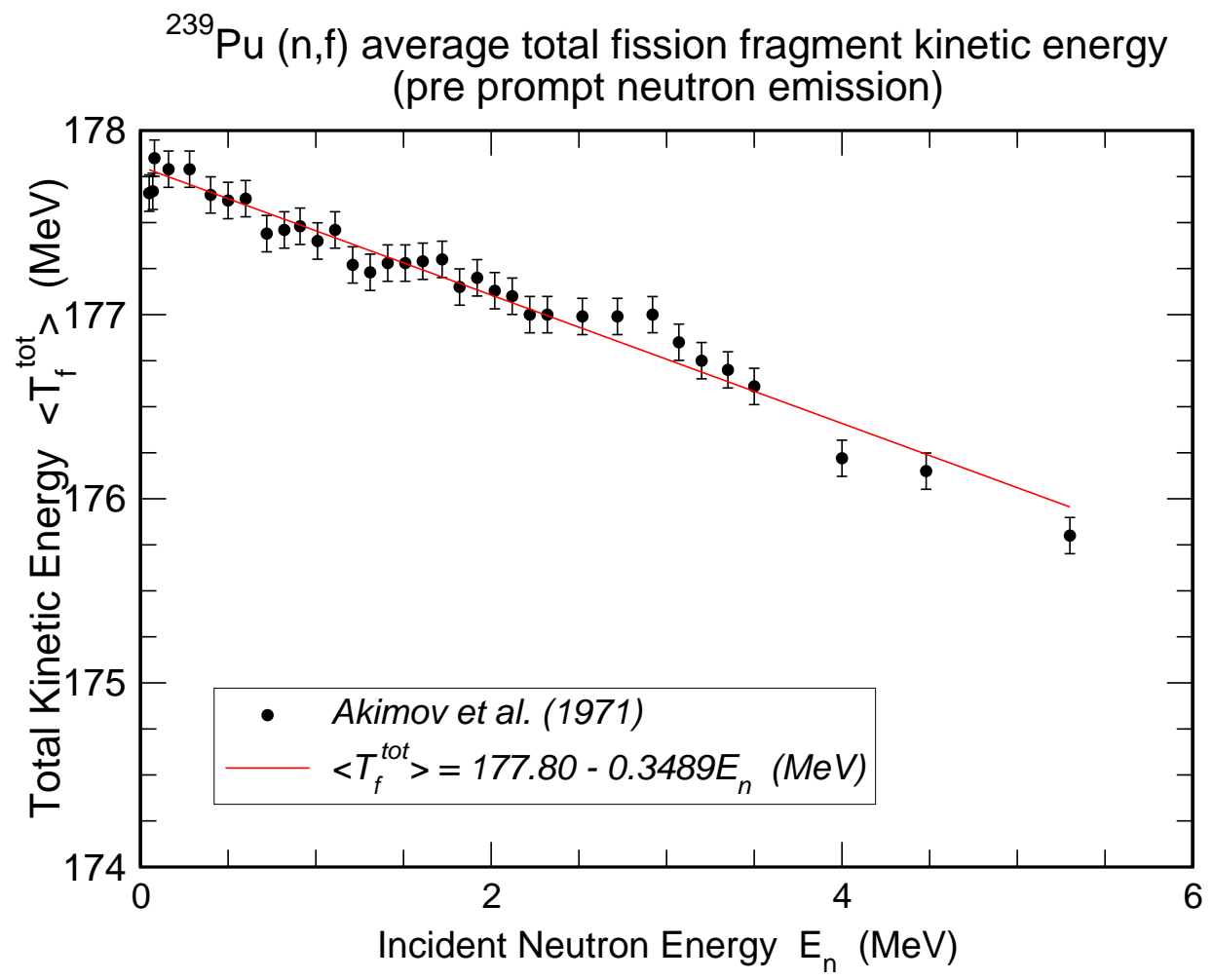

Fig. 6. Average total fission-fragment kinetic energy for the $n\left(E_{n}\right)+{ }^{239} \mathrm{Pu}$ system.

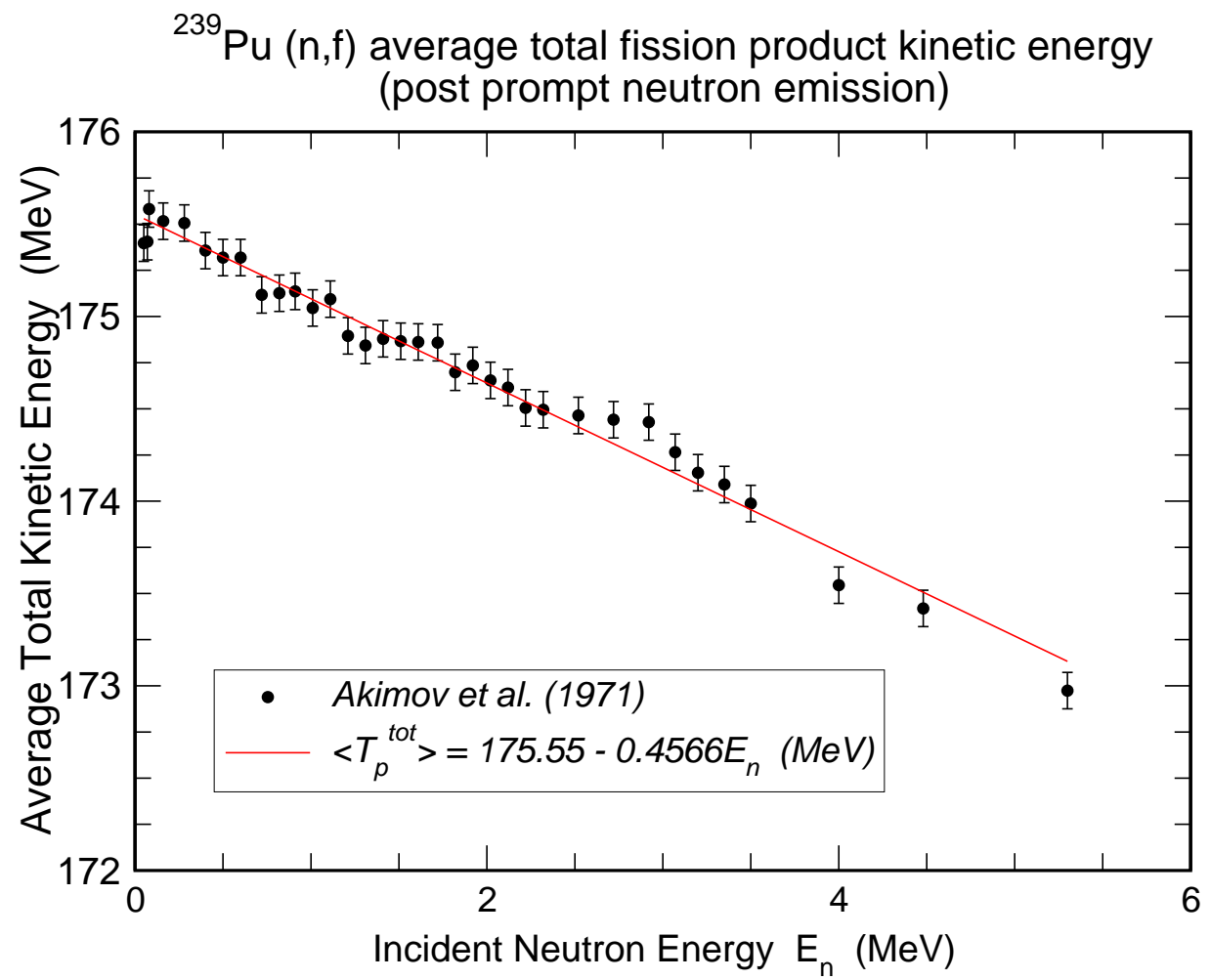

Fig. 7. Average total fission-product kinetic energy for the $\mathrm{n}\left(\mathrm{E}_{\mathrm{n}}\right)+{ }^{239} \mathrm{Pu}$ system. 


\section{For the $\mathbf{n}+{ }^{238} \mathbf{U}$ system:}

$$
\begin{aligned}
\left\langle T_{f}^{t o t}\right\rangle & =(171.7 \pm 0.05)-(0.2396 \pm 0.01) E_{n} \\
& +(0.003434 \pm 0.0004) E_{n}^{2}(\mathrm{MeV}) \\
\left\langle T_{p}^{t o t}\right\rangle & =(169.8 \pm 0.05)-(0.3230 \pm 0.01) E_{n} \\
& +(0.004206 \pm 0.0004) E_{n}^{2}(\mathrm{MeV})
\end{aligned}
$$

The experimental data that we use for the $\mathrm{n}+{ }^{239} \mathrm{Pu}$ system are those of Akimov et al. (1971) [13] shown in Fig. 6 and Fig. 7 for incident neutron energies up to $5.5 \mathrm{MeV}$. Here, the data are not high enough in incident neutron energy to ask whether structure exists near the second-chance fission threshold.

Linear fits appear to be quite adequate for the limited energy range and, strictly, the fits should not be used above about $5.5 \mathrm{MeV}$. Note that in this system we have the steepest drop in the total kinetic energies with increasing incident neutron energy of the three systems under consideration.

\section{For the $\mathbf{n}+{ }^{239} \mathbf{P u}$ system:}

$$
\begin{aligned}
& \left\langle T_{f}^{\text {tot }}\right\rangle=(177.80 \pm 0.03)-(0.3489 \pm 0.02) E_{n}(\mathrm{MeV}) \\
& \left\langle T_{p}^{\text {tot }}\right\rangle=(175.55 \pm 0.03)-(0.4566 \pm 0.02) E_{n}(\mathrm{MeV})
\end{aligned}
$$

For all three of these systems the average total fission-fragment and fissionproduct kinetic energies decrease with increasing incident neutron energy. The reason for this is that the fission-fragment yields for symmetric and nearsymmetric fission are increasing with increasing incident neutron energy [2], but the total kinetic energies are at, or near, a minimum for symmetric and near-symmetric fission, thus decreasing the total kinetic energies with increasing incident neutron energy. This effect has been observed in experiment. See, for example, Fig. 11 of Ref. [14] for the $\mathrm{n}$ (thermal) $+{ }^{235} \mathrm{U}$ system and Fig. 4 of Ref. [15] for the $\mathrm{n}$ (thermal) $+{ }^{239} \mathrm{Pu}$ system. A (speculative) underlying physics reason for the observed effect may be that the charge centers of the nascent fission fragments are slightly farther apart for symmetric fission than they are for asymmetric fission.

The $\mathrm{n}+{ }^{239} \mathrm{Pu}$ system, $\mathrm{Z}=94$, has the largest kinetic energies of the three systems under study due to the Coulomb force, while those of the two U systems, $\mathrm{Z}=92$, are comparable to each other and somewhat less.

The fission product kinetic energies, in addition to being the largest component

of the average total prompt energy release in fission, are the most localized 
in energy deposition having corresponding ranges, for example, of $\sim 5-10$ [microns] in uranium.

\subsection{Average Total Prompt Neutron Emission Energy}

The average total prompt neutron emission energy is equal to the average fission-fragment excitation energy leading to prompt neutron emission $\left\langle E x_{n}^{\text {tot }}\right\rangle$ as given by Eq. (16). It is important to note that this quantity is not equal to the average total prompt fission neutron kinetic energy $\left\langle E_{\text {neut }}^{\text {tot }}\right\rangle$ (to be discussed in Sec. 5) because (a) the portion of the prompt neutron kinetic energy due to the motion of the fission fragments emitting the neutrons has not yet been included and (b) the binding energy $S_{n}$ of the neutron emitted does not contribute to the neutron kinetic energy.

We evaluate Eq. (16) as a function of the incident neutron energy $E_{n}$ as follows: First, the average prompt neutron multiplicities $\bar{\nu}_{p}\left(E_{n}\right)$ are taken from the ENDF evaluations [6] which are based upon experimental data. These are shown for the three systems under study in Fig. 8. Second, the average fissionfragment neutron separation energy $\left\langle S_{n}\right\rangle$ is calculated as one-fourth of the sum of the two two-neutron separation energies for a given binary mass split in a seven-point approximation to the light and heavy mass peaks, as described in Ref. [5], so as to average over two each of the four possible odd-particle configurations. We find $\left\langle S_{n}\right\rangle=4.998 \mathrm{MeV}$ for the $\mathrm{n}+{ }^{235} \mathrm{U}$ system, $\left\langle S_{n}\right\rangle=$ $4.915 \mathrm{MeV}$ for the $\mathrm{n}+{ }^{238} \mathrm{U}$ system, and $\left\langle S_{n}\right\rangle=5.375 \mathrm{MeV}$ for the $\mathrm{n}+$ ${ }^{239} \mathrm{Pu}$ system. For the range of incident neutron energies considered here, it is a reasonable approximation that the $\left\langle S_{n}\right\rangle$ values are constant, independent of the incident energy. Third, the average center-of-mass energies $\langle\varepsilon\rangle$ of the emitted prompt neutrons are calculated with the Los Alamos model [5]:

$$
\langle\varepsilon\rangle=\frac{\left[P_{f_{1}}^{A} \bar{\nu}_{p_{1}}\left\langle\varepsilon_{1}\right\rangle+P_{f_{2}}^{A}\left(\left\langle\xi_{1}\right\rangle+\bar{\nu}_{p_{2}}\left\langle\varepsilon_{2}\right\rangle\right)+P_{f_{3}}^{A}\left(\left\langle\xi_{1}\right\rangle+\left\langle\xi_{2}\right\rangle+\bar{\nu}_{p_{3}}\left\langle\varepsilon_{3}\right\rangle\right)\right]}{\left[P_{f_{1}}^{A} \bar{\nu}_{p_{1}}+P_{f_{2}}^{A}\left(1+\bar{\nu}_{p_{2}}\right)+P_{f_{3}}^{A}\left(2+\bar{\nu}_{p_{3}}\right)\right]}
$$

where, again, $A$ is the mass number of the fissioning compound nucleus, the $P_{f_{i}}^{A}$ are the fission probabilities for $i$ th-chance fission, the $\bar{\nu}_{p_{i}}$ are the average prompt neutron multiplicities for $i$ th-chance fission, the $\xi_{i}$ are the average kinetic energies of the evaporated neutrons prior to fission in 2nd-chance fission $(i=1)$ and 3 rd-chance fission $(i=2)$, and the $\left\langle\varepsilon_{i}\right\rangle$ are the average center-ofmass neutron energies for $i$ th-chance fission.

Equation (24) has been evaluated as a function of incident neutron energy for the three systems under study and the results are shown in Fig. 9. One 
sees that the values of $\langle\varepsilon\rangle$ generally increase with increasing $E_{n}$, but decrease near $6 \mathrm{MeV}$ and $13 \mathrm{MeV}$ which are the approximate thresholds for 2nd- and 3rd-chance fission where the emission of 1 and 2 neutrons, respectively, prior to fission, reduce the fragment excitation energy available for neutron and gamma emission and, correspondingly, reduce $\langle\varepsilon\rangle$.

Note that the structure observed below $\sim 3 \mathrm{MeV}$ in the $\mathrm{n}+{ }^{235} \mathrm{U}$ system is due to fits of the Los Alamos model to experimental spectra measured at these energies. It is clear from Figs. 8 and 9 that the $\mathrm{n}+{ }^{239} \mathrm{Pu}$ system is the hottest of the three systems in terms of both the number of neutrons emitted and their energy, with the $n+{ }^{235} \mathrm{U}$ and $\mathrm{n}+{ }^{238} \mathrm{U}$ systems very similar, but somewhat larger neutron emission energies for $\mathrm{n}+{ }^{235} \mathrm{U}$.

Using the preceding information, Eq. (16) for the average fission-fragment excitation energy leading to prompt neutron emission (average total prompt neutron emission energy) can be evaluated for the three systems under study.

The results are shown in Fig. 10 which indicate an approximately linear dependence upon the incident neutron energy $E_{n}$ and, again, more neutron emission for the $\mathrm{Pu}$ system and less, but comparable, emission for the two U systems.

Linear fits to the three calculations shown in Fig. 10 are illustrated in Figs. 11 - 13 together with the calculated points from Eq. (16) wherein the values of $\bar{\nu}_{p}\left(E_{n}\right)$ are taken from ENDF.

For the $\mathbf{n}+{ }^{235} \mathbf{U}$ system:

$$
\left\langle E x_{n}^{t o t}\right\rangle=14.59+0.9772 E_{n}(M e V)
$$

For the $\mathbf{n}+{ }^{238} \mathbf{U}$ system:

$$
\left\langle E x_{n}^{t o t}\right\rangle=14.11+0.9839 E_{n}(M e V)
$$

For the $\mathbf{n}+{ }^{239} \mathbf{P u}$ system:

$$
\left\langle E x_{n}^{t o t}\right\rangle=19.23+1.0707 E_{n}(M e V)
$$




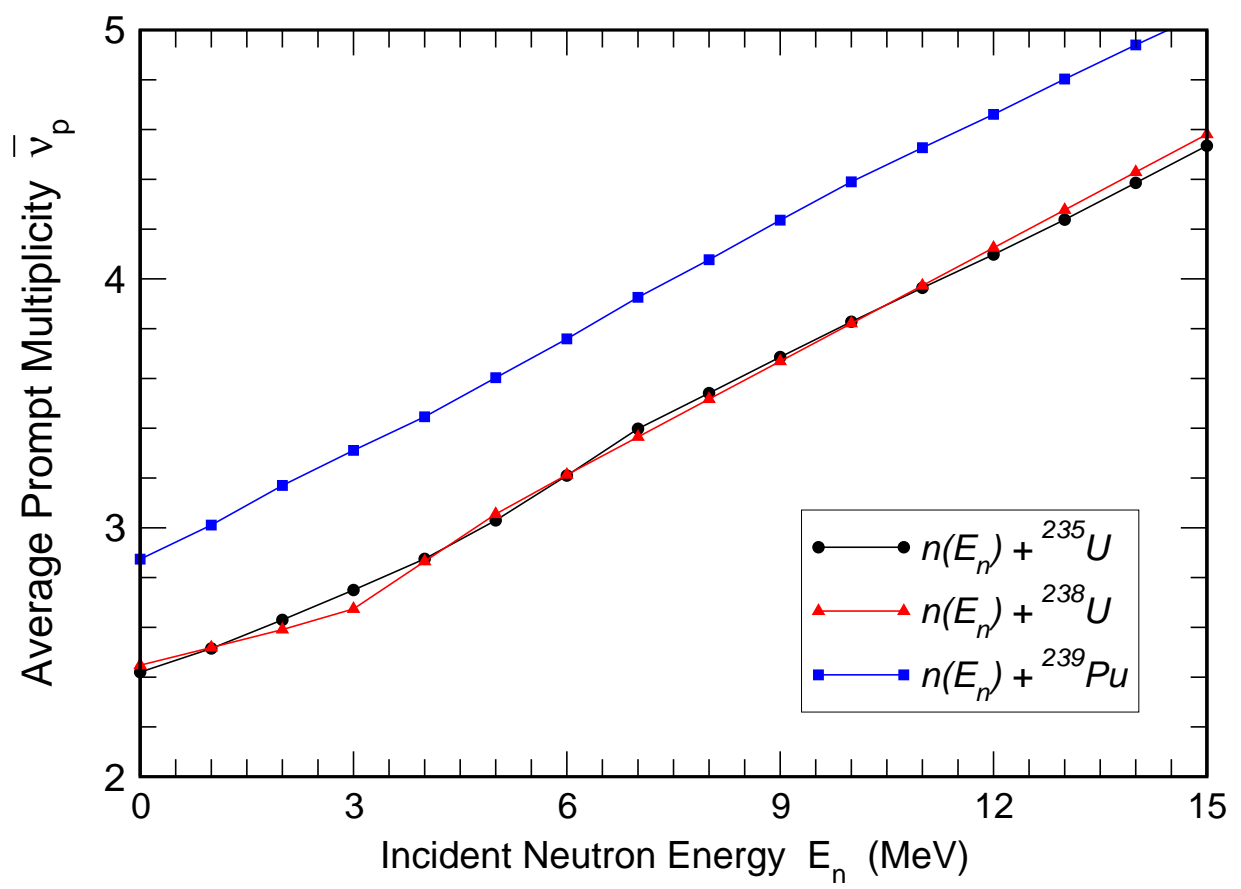

Fig. 8. Average prompt fission neutron multiplicity $\bar{\nu}_{p}$ for three systems from ENDF evaluated data (line segments are to guide the eye).

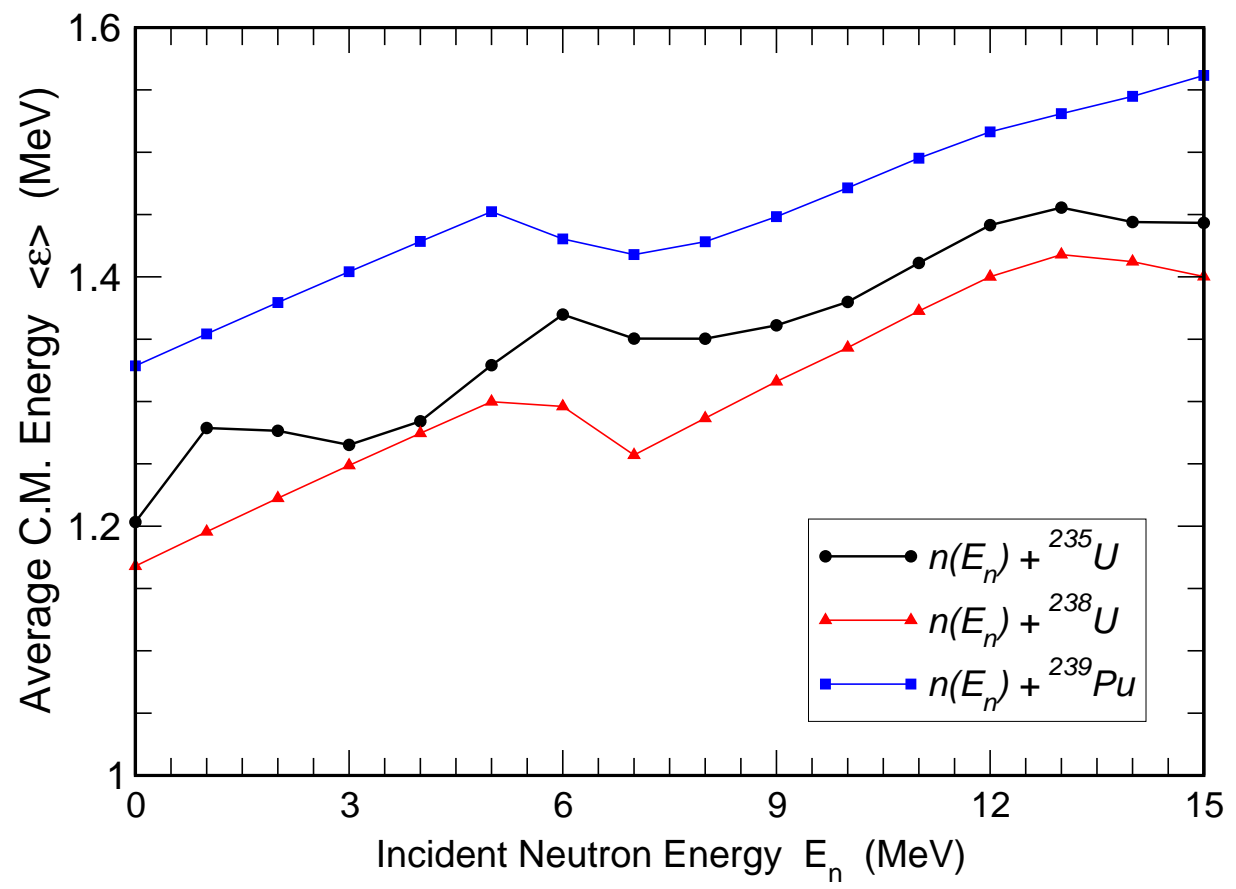

Fig. 9. Prompt fission neutron spectrum average center-of-mass energy $\langle\varepsilon\rangle$ for three systems calculated with the Los Alamos model (line segments are to guide the eye). 


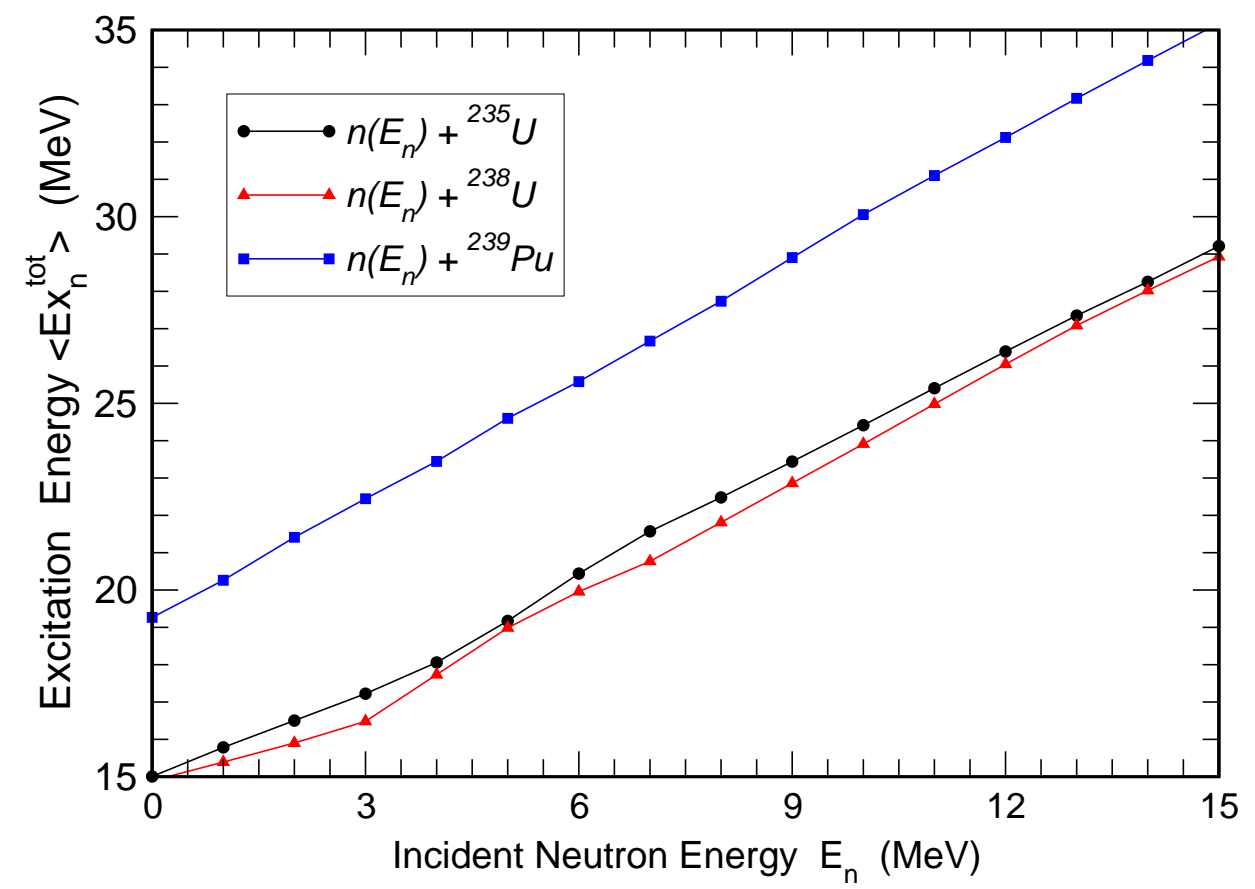

Fig. 10. Fission fragment excitation energy $\left\langle E x_{n}^{t o t}\right\rangle$ leading to prompt neutron emission for three systems from the Los Alamos model and ENDF (line segments are to guide the eye).

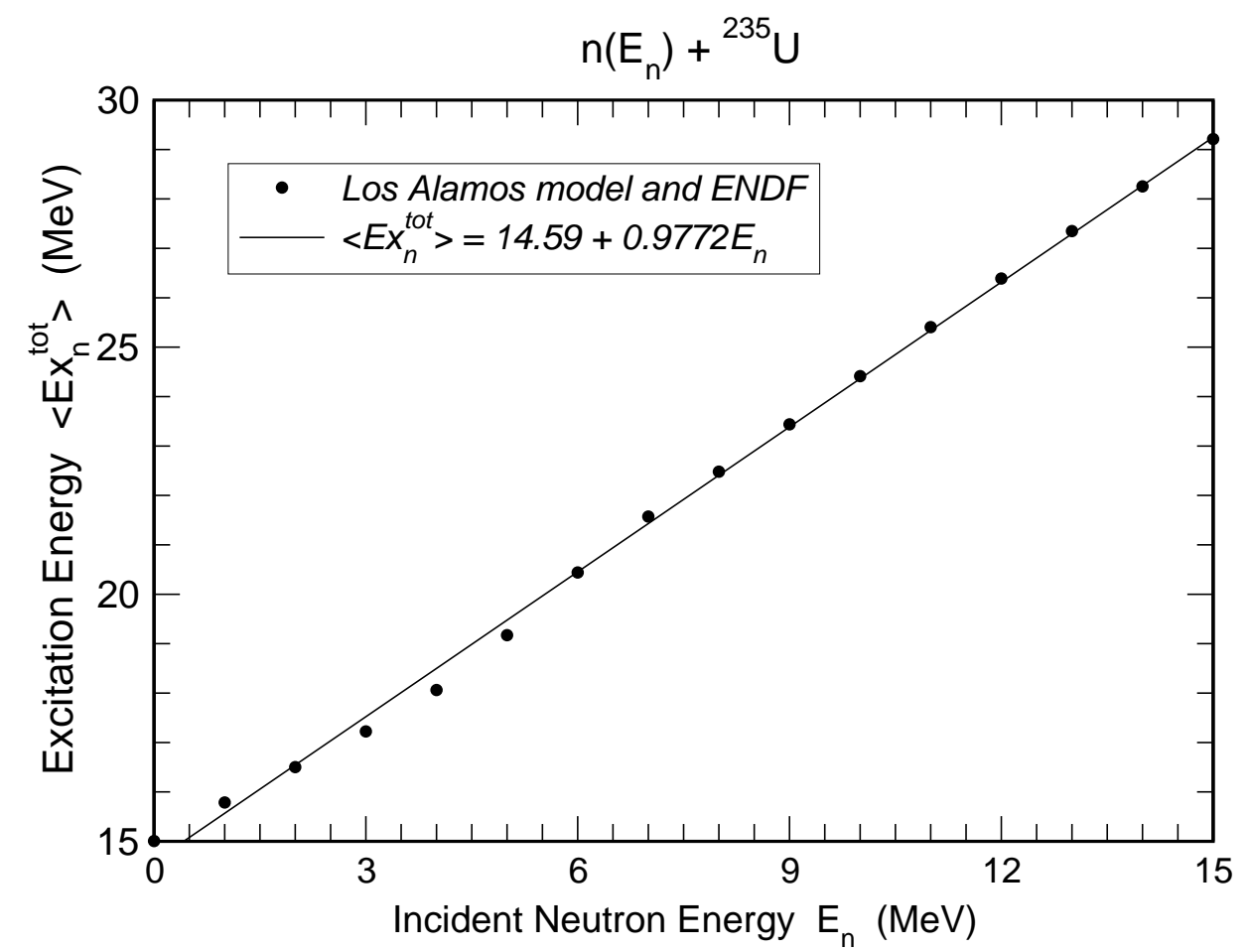

Fig. 11. Fission fragment excitation energy $\left\langle E x_{n}^{t o t}\right\rangle$ leading to prompt neutron emission in the $n\left(E_{n}\right)+{ }^{235} U$ system. 


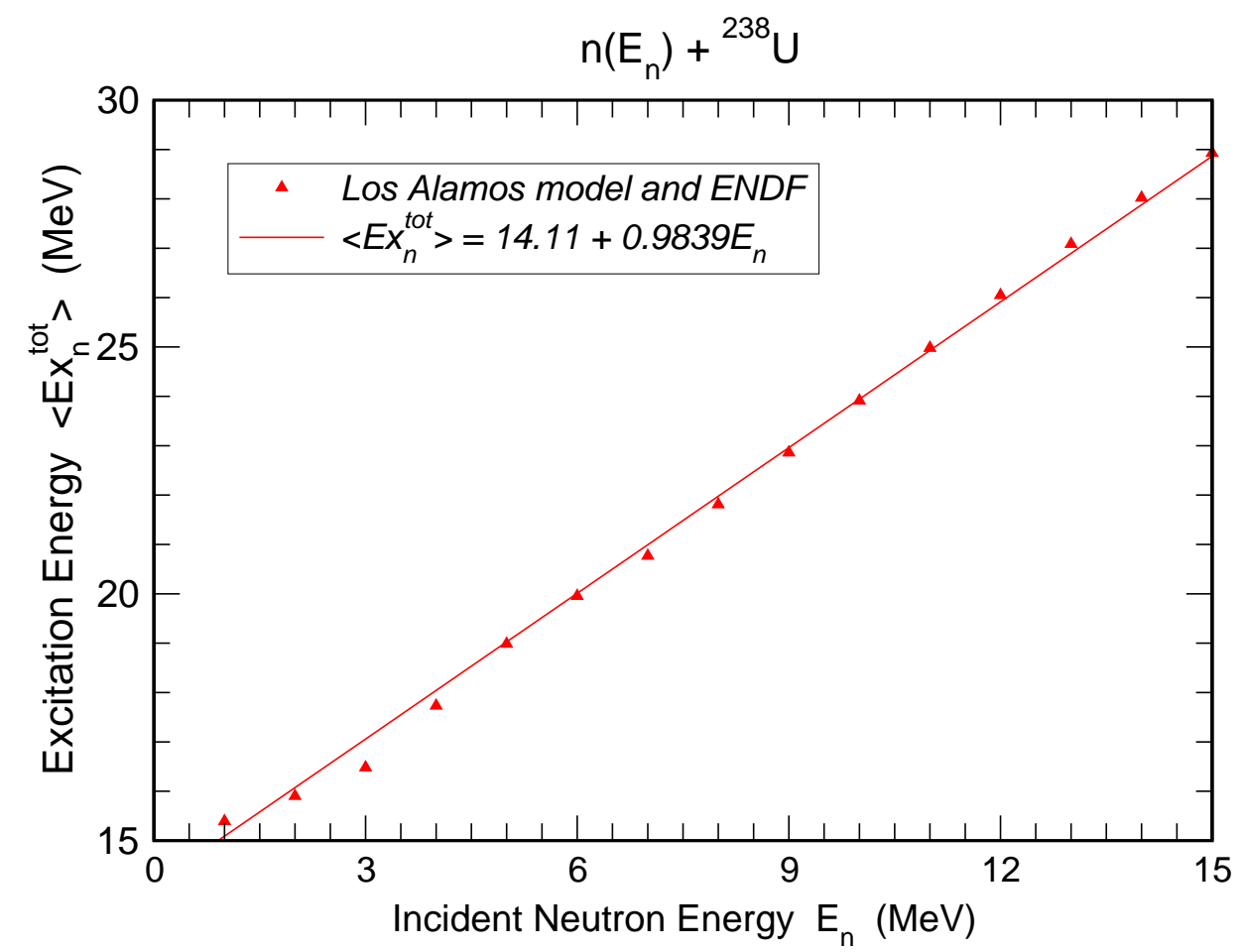

Fig. 12. Fission fragment excitation energy $\left\langle E x_{n}^{t o t}\right\rangle$ leading to prompt neutron emission in the $\mathrm{n}\left(\mathrm{E}_{\mathrm{n}}\right)+{ }^{238} \mathrm{U}$ system.

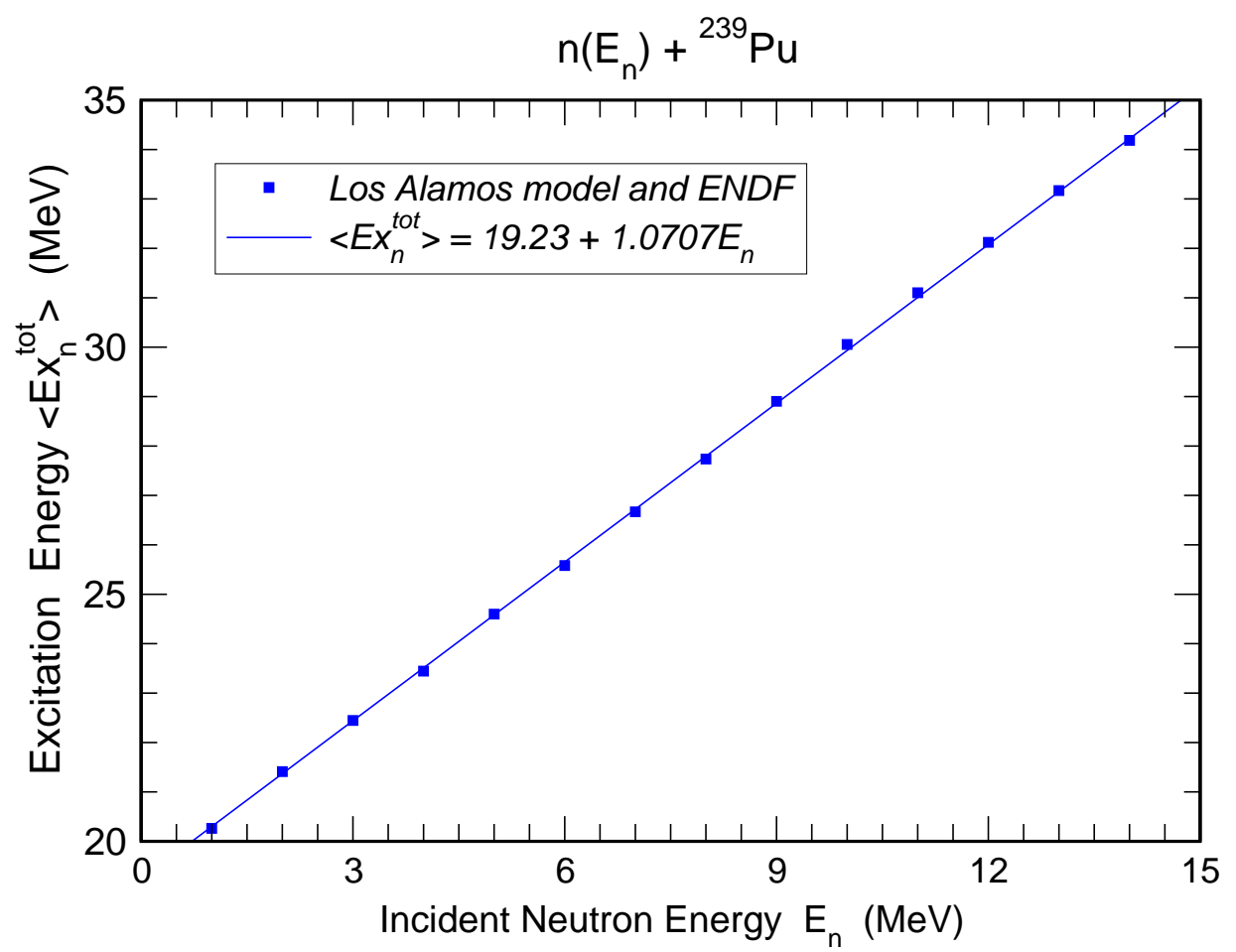

Fig. 13. Fission fragment excitation energy $\left\langle E x_{n}^{t o t}\right\rangle$ leading to prompt neutron emission in the $\mathrm{n}\left(\mathrm{E}_{\mathrm{n}}\right)+{ }^{239} \mathrm{Pu}$ system. 


\subsection{Average Total Prompt Gamma Emission Energy}

The experimental data that we use for the $\mathrm{n}+{ }^{235} \mathrm{U}$ system are those of Frehaut et al. (1982) [16] which are ratio measurements to the average total prompt gamma emission energy for ${ }^{252} \mathrm{Cf}(\mathrm{sf})$. These ratio data have been converted back to absolute units by using the average of three measurements for ${ }^{252} \mathrm{Cf}$ reported in the Hoffman and Hoffman review [17]. The converted Frehaut et al. data are shown in Fig. 14 together with a linear fit to the data. There is nonlinear structure in these data, but for our present purposes we use the linear approximation.

For the $\mathbf{n}+{ }^{235} \mathbf{U}$ system:

$$
\left\langle E_{\gamma}^{\text {tot }}\right\rangle=(6.600 \pm 0.03)+(0.0777 \pm 0.004) E_{n}(\mathrm{MeV})
$$

We have no experimental values for the average total prompt gamma emission energy in the $\mathrm{n}+{ }^{238} \mathrm{U}$ system. Therefore, an empirical approach is used, namely, a linear assumption with $\bar{\nu}_{p}\left(E_{n}\right)$ is made (based upon the Frehaut et al. [16] measurements) with the zero (thermal) energy value taken from the $A$-dependent fit by Hoffman and Hoffman [17], and the slope taken from that inferred by Frehaut et al. from the $\mathrm{n}+{ }^{237} \mathrm{~Np}$ measurements that they performed. Note, however, that we use the lower experimental limit of their inferred slope because the $\mathrm{n}+{ }^{237} \mathrm{~Np}$ system is hotter than that of $\mathrm{n}+{ }^{238} \mathrm{U}$. The resulting data points are labeled "Empirical (2004)" in Fig. 15 together with a linear fit in incident neutron energy.

For the $\mathbf{n}+{ }^{238} \mathbf{U}$ system:

$$
\left\langle E_{\gamma}^{\text {tot }}\right\rangle=6.6800+0.1239 E_{n}(\mathrm{MeV})
$$

Experimental values exist for the average total prompt gamma emission energy for the $\mathrm{n}+{ }^{239} \mathrm{Pu}$ system, but only for thermal neutron energy: Pleasonton (1973) [18] measured a value of $6.73 \pm 0.35 \mathrm{MeV}$ for the thermal case. Direct measurements for greater neutron energy do not appear to exist.

Consequently, we employ an evaluation by Fort (1994) [19] which is based upon systematics with respect to the measurements by Frehaut et al. [16] on nearby actinides and upon multichance fission probabilities from the Japanese Nuclear Data Center. This evaluation is shown as the points appearing in Fig. 16 together with a quadratic fit in the incident neutron energy. 


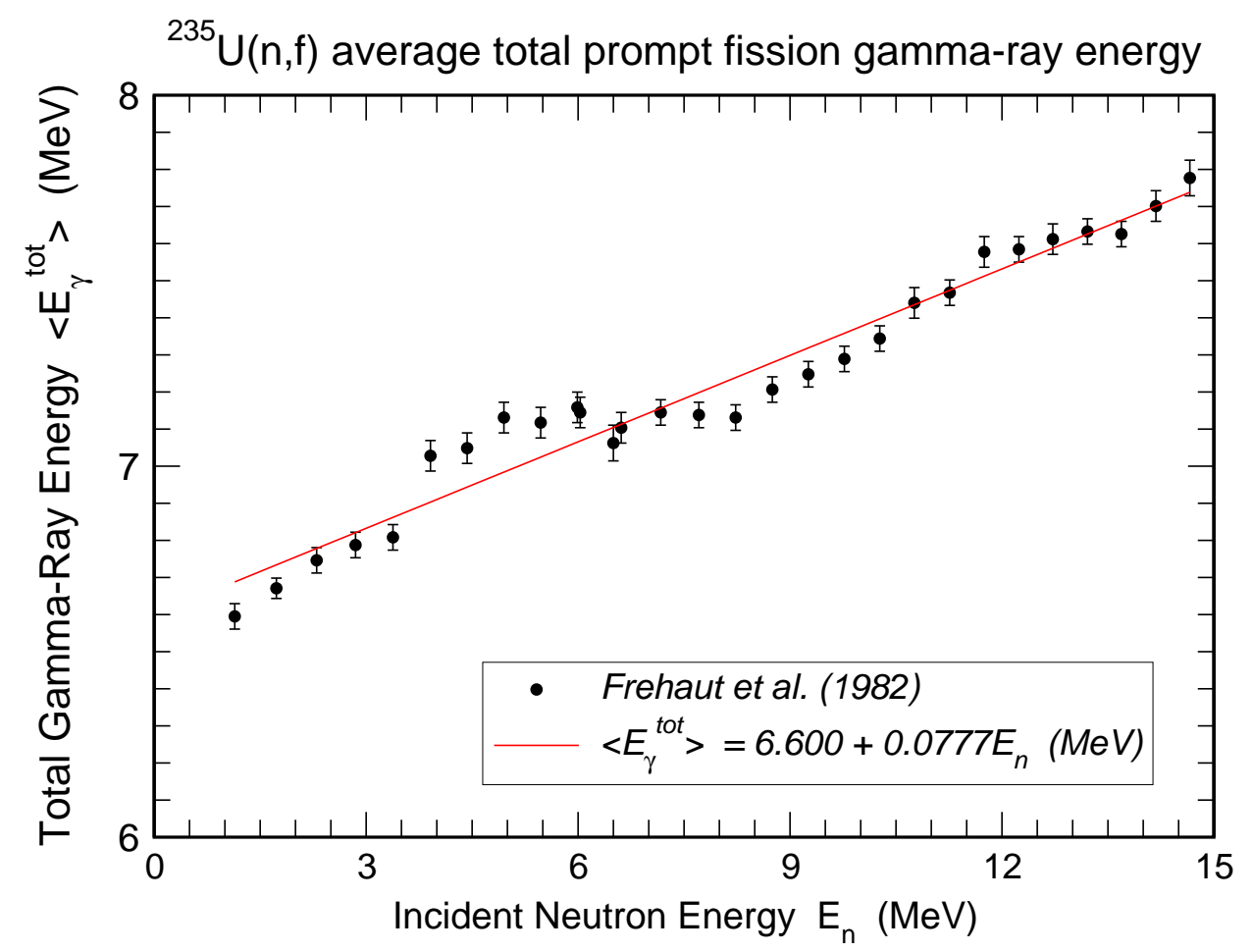

Fig. 14. Average total prompt fission gamma-ray energy $\left\langle E_{\gamma}^{\text {tot }}\right\rangle$ for the $n\left(E_{n}\right)+{ }^{235} U$ system.

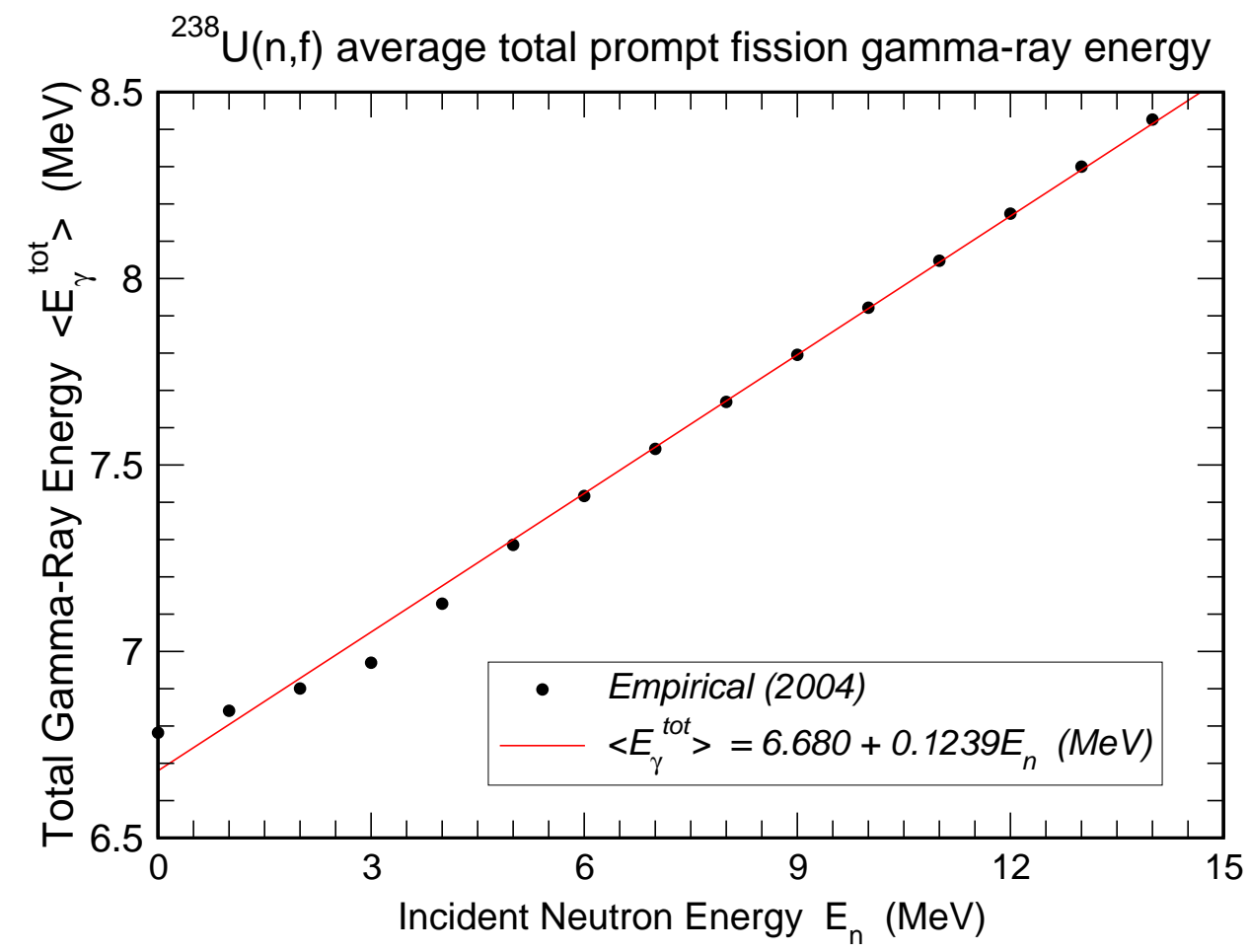

Fig. 15. Average total prompt fission gamma-ray energy $\left\langle E_{\gamma}^{\text {tot }}\right\rangle$ for the $n\left(E_{n}\right)+{ }^{238} U$ system. 


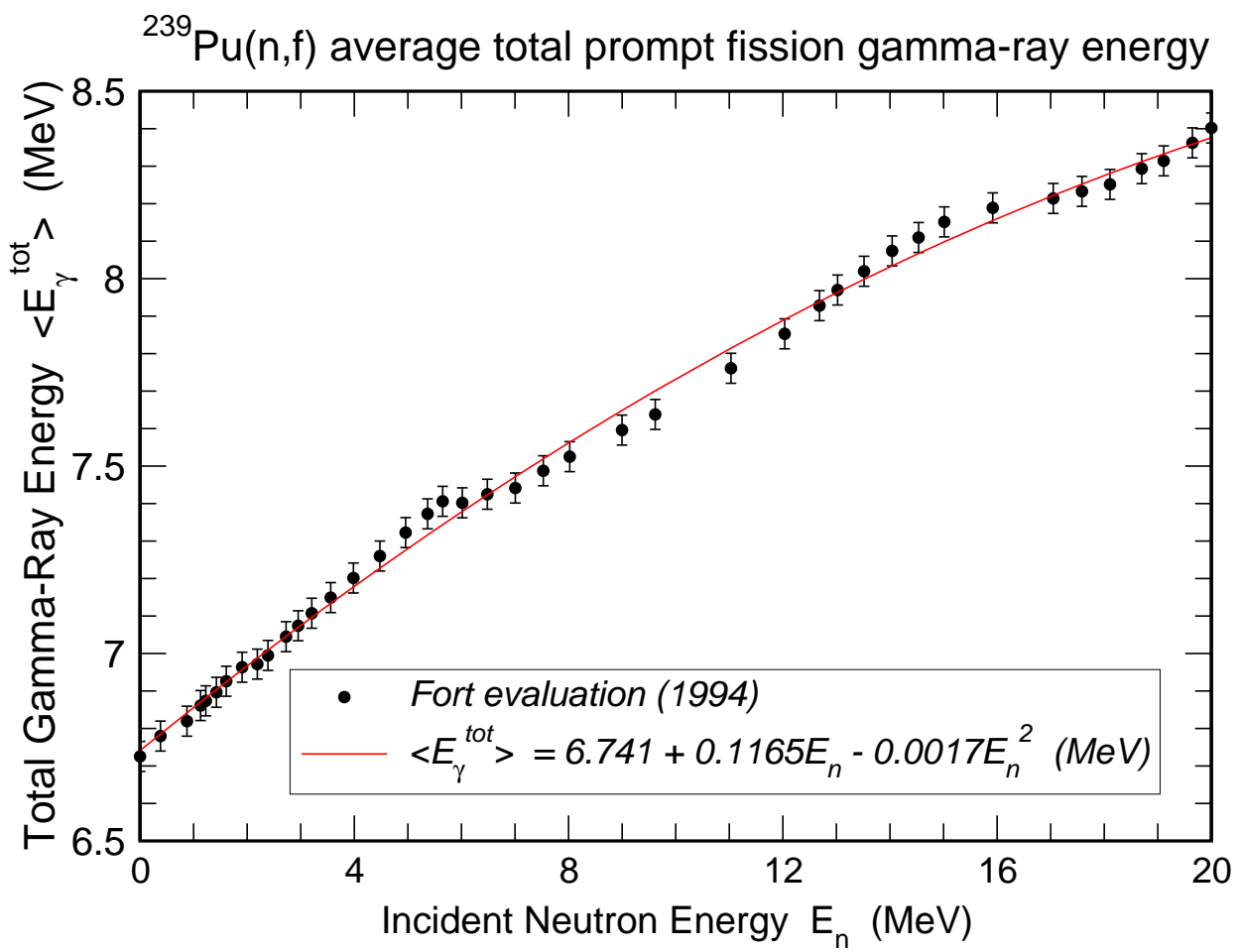

Fig. 16. Average total prompt fission gamma-ray energy $\left\langle E_{\gamma}^{\text {tot }}\right\rangle$ for the $n\left(E_{n}\right)+$ ${ }^{239} \mathrm{Pu}$ system.

For the $\mathbf{n}+{ }^{239} \mathbf{P u}$ system:

$$
\begin{aligned}
\left\langle E_{\gamma}^{\text {tot }}\right\rangle & =(6.741 \pm 0.02)+(0.1165 \pm 0.004) E_{n} \\
& -(0.0017 \pm 0.0002) E_{n}^{2}(\mathrm{MeV})
\end{aligned}
$$

\section{Average Total Prompt Fission Energy Release}

Equation (17) for the average total prompt energy release in fission can now be evaluated for the three systems under study:

For the $\mathbf{n}+{ }^{235} \mathbf{U}$ system one substitutes Eq. (18), Eq. (25), and Eq. (28) into Eq. (17), together with the value $B_{n}=6.546 \mathrm{MeV}$ for this system, to obtain

$$
\left\langle E_{r}\right\rangle=185.6-0.0995 E_{n}(\mathrm{MeV})
$$

For the $\mathbf{n}+{ }^{238} \mathbf{U}$ system one substitutes Eq. (20), Eq. (26), and Eq. (29) into Eq. (17), together with the value $B_{n}=4.806 \mathrm{MeV}$ for this system, to 
obtain

$$
\left\langle E_{r}\right\rangle=187.7-0.1318 E_{n}+0.0034 E_{n}^{2}(\mathrm{MeV})
$$

For the $\mathbf{n}+{ }^{239} \mathbf{P u}$ system one substitutes Eq. (22), Eq. (27), and Eq. (30) into Eq. (17), together with the value $B_{n}=6.534 \mathrm{MeV}$ for this system, to obtain

$$
\left\langle E_{r}\right\rangle=197.2-0.1617 E_{n}-0.0017 E_{n}^{2}(\mathrm{MeV})
$$

The calculated average total prompt fission energy release for $\left\langle E_{r}\right\rangle$ the three systems is shown in Fig. 17. There are two major features in this figure. First, the prompt energy release for the neutron-induced fission of ${ }^{239} \mathrm{Pu}$ is about 10 $\mathrm{MeV}$ greater than that of the two isotopes of $\mathrm{U}$, over the entire energy range of $15 \mathrm{MeV}$, and the prompt energy release for the neutron-induced fission of ${ }^{238} \mathrm{U}$ is about $2 \mathrm{MeV}$ greater than that of ${ }^{235} \mathrm{U}$, over the same energy range. Second, the prompt energy release decreases with increasing incident neutron energy for all three of the systems under study, which is contrary to intuition.

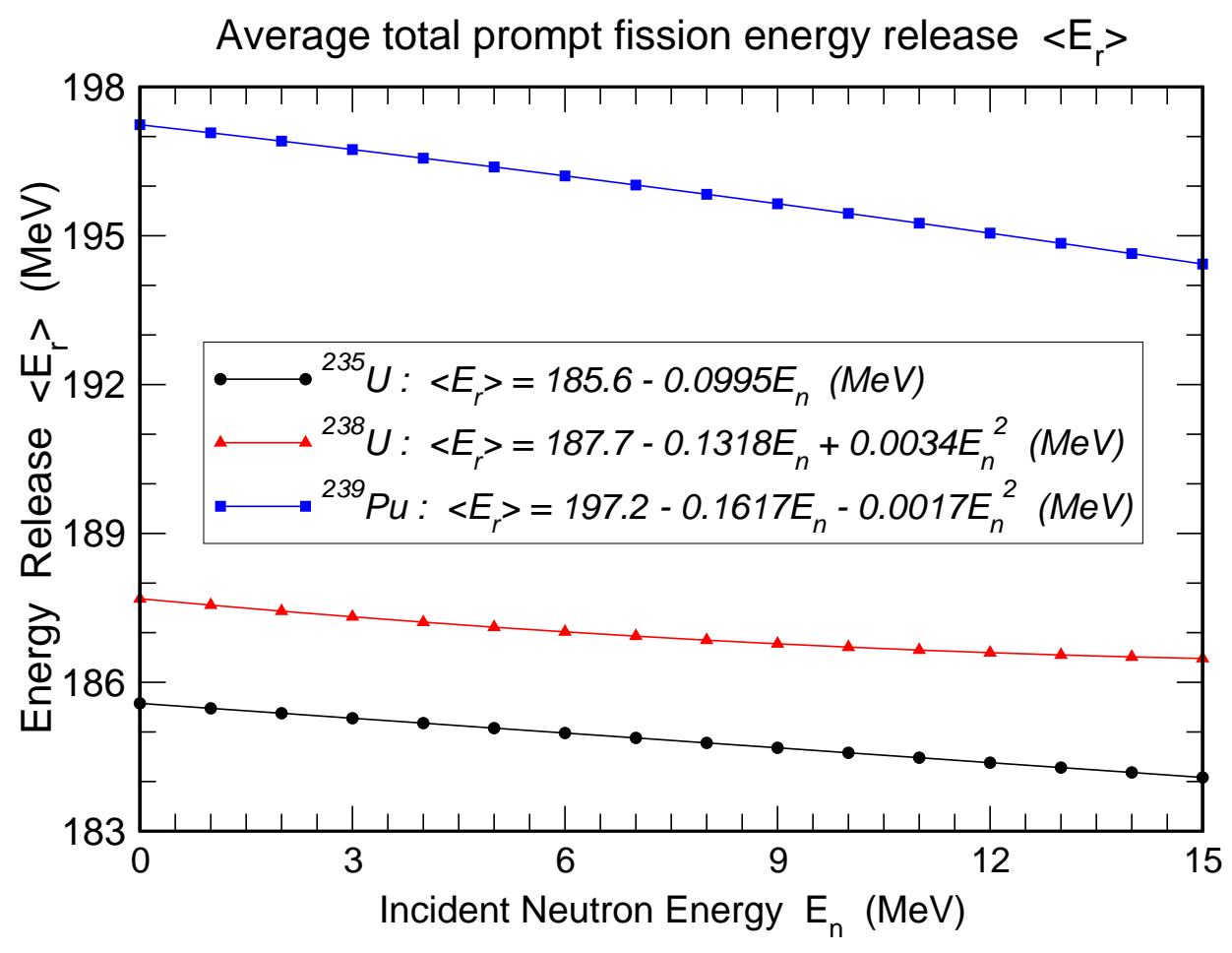

Fig. 17. Average total prompt fission energy release $\left\langle E_{\mathrm{r}}\right\rangle$ for three systems. 
As already pointed out, this behavior is primarily due to the facts that symmetric fission increases with increasing neutron energy and that the total kinetic energy for symmetric fission is significantly less than the total kinetic energy for the more probable asymmetric fission. At the same time, however, the average prompt fission neutron multiplicities as a function of fission-fragment mass, $\bar{\nu}_{p}\left(A_{f}\right)$, are peaked for symmetric fision. Note that for far asymmetric fission (which is relatively infrequent) the total kinetic energy is also significantly reduced.

In the next section we examine the average total prompt energy deposition in the medium in which the fission event occurs, for the three systems under study.

\section{Average Total Prompt Fission Energy Deposition}

The average total prompt fission energy deposition in the medium for binary fission $\left\langle E_{d}\right\rangle$ is defined as the average total prompt fission energy release in binary fission $\left\langle E_{r}\right\rangle$ plus the total energy brought to the fission event by the particle inducing the fission [the quantity $\left(E_{n}+B_{n}\right)$ for neutron-induced fission] minus the average total binding energy of the prompt neutrons emitted by the fission fragments $\bar{\nu}_{p}\left\langle S_{n}\right\rangle$ [which is not deposited in the medium]. With this definition and Eq. (17) we obtain

$$
\begin{aligned}
\left\langle E_{d}\right\rangle & =\left\langle E_{r}\right\rangle+\left(E_{n}+B_{n}\right)-\bar{\nu}_{p}\left\langle S_{n}\right\rangle \\
& =\left\langle T_{f}^{t o t}\right\rangle+\bar{\nu}_{p}\langle\varepsilon\rangle+\left\langle E_{\gamma}^{t o t}\right\rangle .
\end{aligned}
$$

We wish to express Eq. (35) in terms of laboratory observables in the medium. Therefore, note that Eq. (14) is of the form $\left\langle T_{p}^{t o t}\right\rangle=\left\langle T_{f}^{t o t}\right\rangle[1-x]$ where $x$ is a small quantity. Solving Eq. (14) for $\left\langle T_{f}^{t o t}\right\rangle$ and performing an expansion of $1 /[1-x]$ yields

$$
\left\langle T_{f}^{t o t}\right\rangle=\left\langle T_{p}^{t o t}\right\rangle\left[1+\frac{\bar{\nu}_{p}}{2 A}\left(\frac{\left\langle A_{H}\right\rangle}{\left\langle A_{L}\right\rangle}+\frac{\left\langle A_{L}\right\rangle}{\left\langle A_{H}\right\rangle}\right)\right]
$$

Inserting Eq. (36) into Eq. (35) and rearranging terms gives

$$
\left\langle E_{d}\right\rangle=\left\langle T_{p}^{t o t}\right\rangle+\left\langle E_{\text {neut }}^{\text {tot }}\right\rangle+\left\langle E_{\gamma}^{\text {tot }}\right\rangle
$$

with $\left\langle E_{\text {neut }}^{\text {tot }}\right\rangle$ the average total prompt fission neutron kinetic energy in the laboratory system given by 


$$
\begin{aligned}
\left\langle E_{\text {neut }}^{\text {tot }}\right\rangle & =\bar{\nu}_{p}\left[\frac{1}{2}\left(\frac{\left\langle A_{H}\right\rangle}{\left\langle A_{L}\right\rangle} \frac{\left\langle T_{f}^{t o t}\right\rangle}{A}+\frac{\left\langle A_{L}\right\rangle}{\left\langle A_{H}\right\rangle} \frac{\left\langle T_{f}^{t o t}\right\rangle}{A}\right)+\langle\varepsilon\rangle\right] \\
& =\bar{\nu}_{p}\left[\frac{1}{2}\left(\left\langle E_{f}^{L}\right\rangle+\left\langle E_{f}^{H}\right\rangle\right)+\langle\varepsilon\rangle\right] \\
& =\bar{\nu}_{p}\langle E\rangle
\end{aligned}
$$

in which the prompt fission neutron spectrum average laboratory energy $\langle E\rangle$ is given by the Los Alamos model [5]

$$
\langle E\rangle=\frac{1}{2}\left(\left\langle E_{f}^{L}\right\rangle+\left\langle E_{f}^{H}\right\rangle\right)+\langle\varepsilon\rangle,
$$

and the approximation has been made in Eq. (38) that $\left\langle T_{p}^{\text {tot }}\right\rangle / A$ can be replaced by $\left\langle T_{f}^{\text {tot }}\right\rangle / A$ given that the $\left\langle A_{L, H}\right\rangle$ are not unique, but instead averages, and given that $\langle\varepsilon\rangle$ is the dominant term in the square brackets. In Eqs. (39) and (41) the quantities $\left\langle E_{f}^{L}\right\rangle$ and $\left\langle E_{f}^{H}\right\rangle$ are the average kinetic energies per nucleon of the moving light and heavy fission fragments, respectively, and are the quantities not included in $\left\langle E x_{n}^{t o t}\right\rangle$ as discussed in the beginning of Sec. 3.2.

Note that all averaged quantities appearing in Eqs. (37 - 41) depend upon the incident neutron energy $E_{n}$ which has been suppressed for brevity. We use Eq. (37) for the average total prompt fission energy deposition for the remainder of this paper.

The evaluation of Eq. (40) for the three systems of interest is performed using the $\bar{\nu}_{p}$ values from ENDF [6] shown in Fig. 8 and prompt fission neutron spectrum average laboratory energies $\langle E\rangle$ calculated for multi-chance fission with the Los Alamos model [5] :

$$
\langle E\rangle=\frac{\left[P_{f_{1}}^{A} \bar{\nu}_{p_{1}}\left\langle E_{1}\right\rangle+P_{f_{2}}^{A}\left(\left\langle\xi_{1}\right\rangle+\bar{\nu}_{p_{2}}\left\langle E_{2}\right\rangle\right)+P_{f_{3}}^{A}\left(\left\langle\xi_{1}\right\rangle+\left\langle\xi_{2}\right\rangle+\bar{\nu}_{p_{3}}\left\langle E_{3}\right\rangle\right)\right]}{\left[P_{f_{1}}^{A} \bar{\nu}_{p_{1}}+P_{f_{2}}^{A}\left(1+\bar{\nu}_{p_{2}}\right)+P_{f_{3}}^{A}\left(2+\bar{\nu}_{p_{3}}\right)\right]}
$$

where the $\left\langle E_{i}\right\rangle$ are the average laboratory neutron energies for ith-chance fission and all other quantities are defined as for Eq. (24).

The prompt fission neutron spectrum average laboratory energies $\langle E\rangle$ calculated with Eq. (42) for the three systems are shown in Fig. 18. Just as with the average center-of-mass energies $\langle\varepsilon\rangle$, the values of $\langle E\rangle$ decrease near $6 \mathrm{MeV}$ and $13 \mathrm{MeV}$, the approximate thresholds for 2nd- and 3rd-chance fission, and for the same reasons. The product of these two quantities $\left\langle E_{\text {neut }}^{\text {tot }}\right\rangle$ is shown as a function of incident neutron energy in Fig. 19 for the three systems of 
interest. Some evidence of multiple-chance fission threshold structure is still present.

Again, for our present purposes, we perform linear fits to the values of the average total prompt fission neutron kinetic energies $\left\langle E_{\text {neut }}^{\text {tot }}\right\rangle$ shown in Fig. 19 for the three systems. The results are shown together with the calculated values in Figs. 20, 21, and 22.

For the $\mathbf{n}+{ }^{235} \mathbf{U}$ system:

$$
\left\langle E_{\text {neut }}^{\text {tot }}\right\rangle=4.838+0.3004 E_{n}(\mathrm{MeV})
$$

For the $\mathbf{n}+{ }^{238} \mathbf{U}$ system:

$$
\left\langle E_{\text {neut }}^{\text {tot }}\right\rangle=4.558+0.3070 E_{n}(M e V)
$$

For the $\mathbf{n}+{ }^{239} \mathbf{P u}$ system:

$$
\left\langle E_{\text {neut }}^{\text {tot }}\right\rangle=6.128+0.3428 E_{n}(\mathrm{MeV})
$$

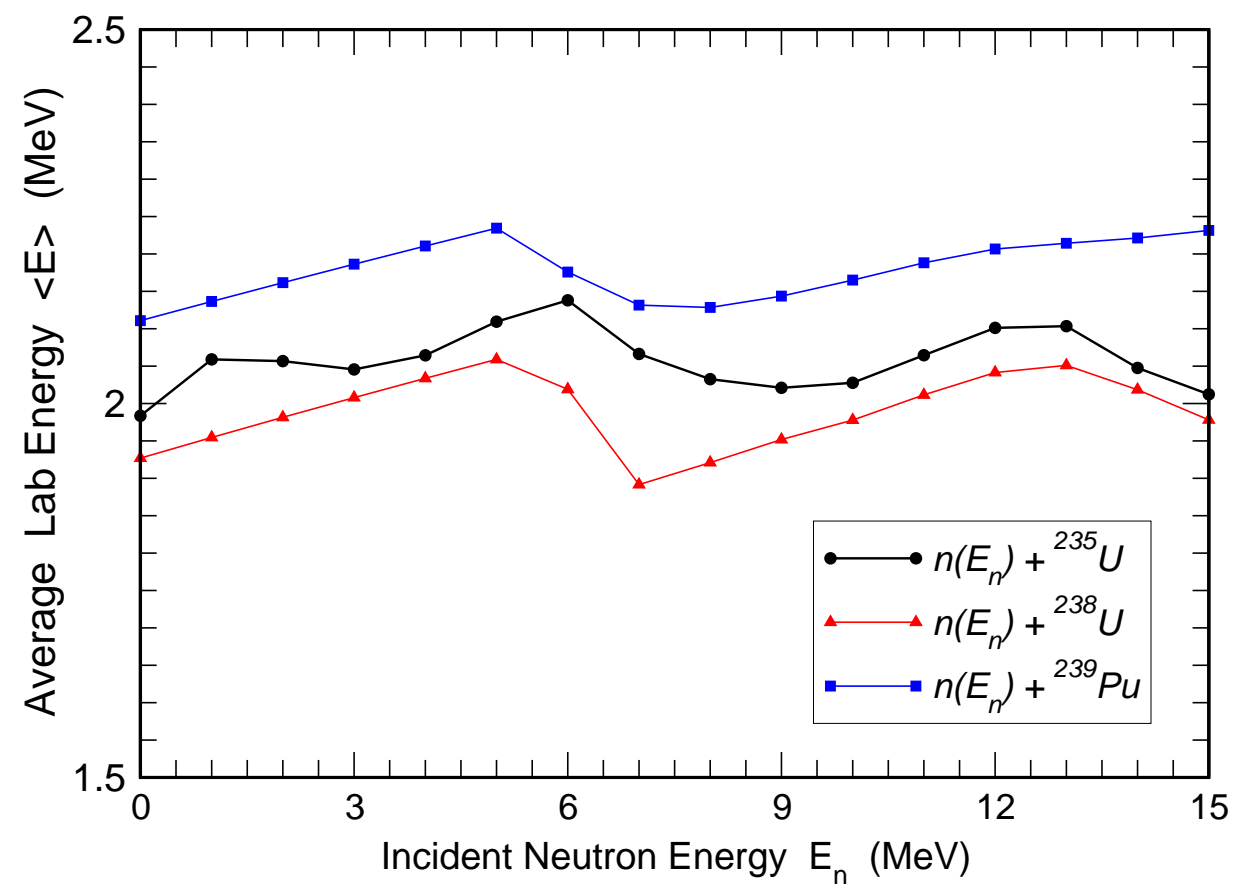

Fig. 18. Prompt fission neutron spectrum average laboratory energy $\langle E\rangle$ for three systems calculated with the Los Alamos model (line segments are to guide the eye). 


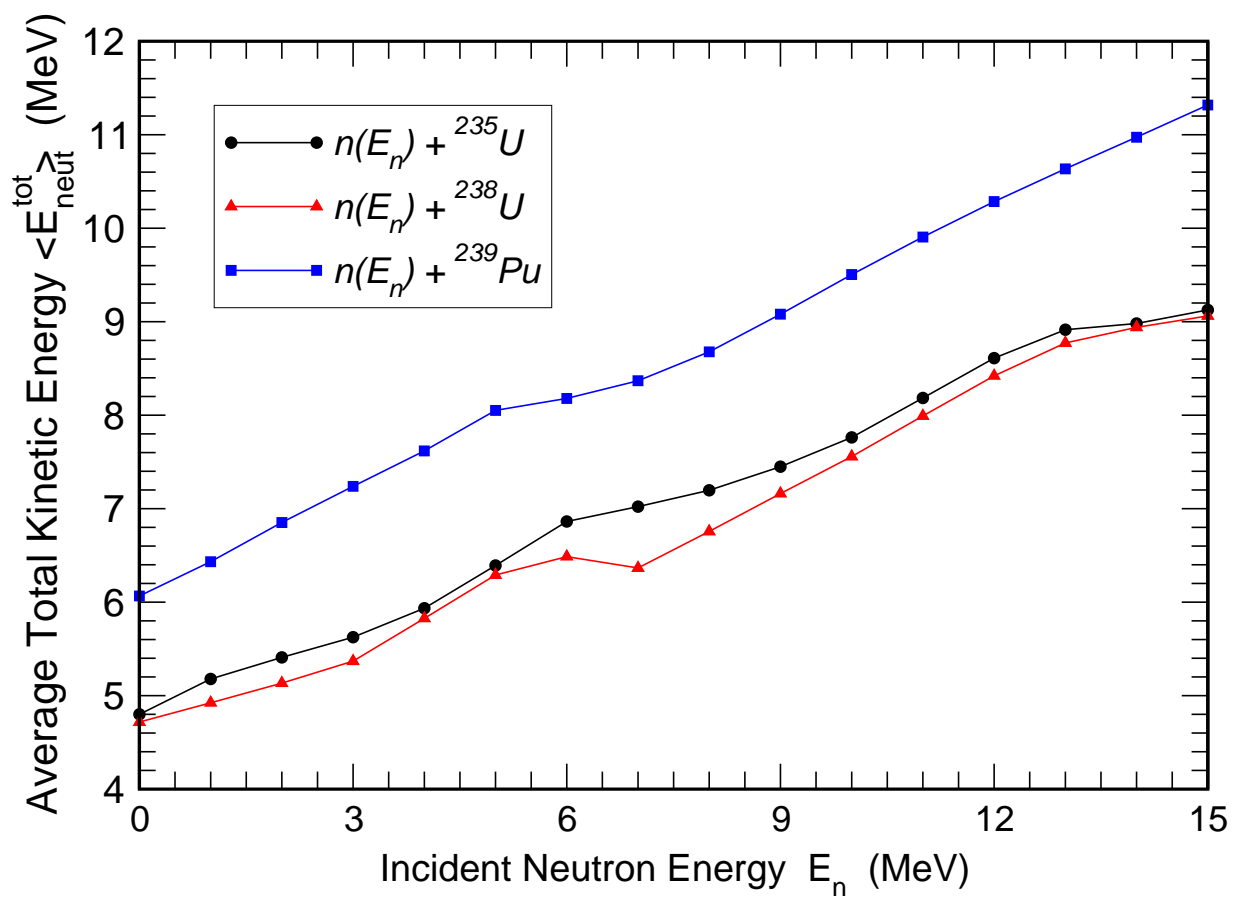

Fig. 19. Average total prompt fission neutron kinetic energy $\left\langle\mathrm{E}_{\text {neut }}^{\text {tot }}\right\rangle$ for three systems from the Los Alamos model and ENDF (line segments are to guide the eye).

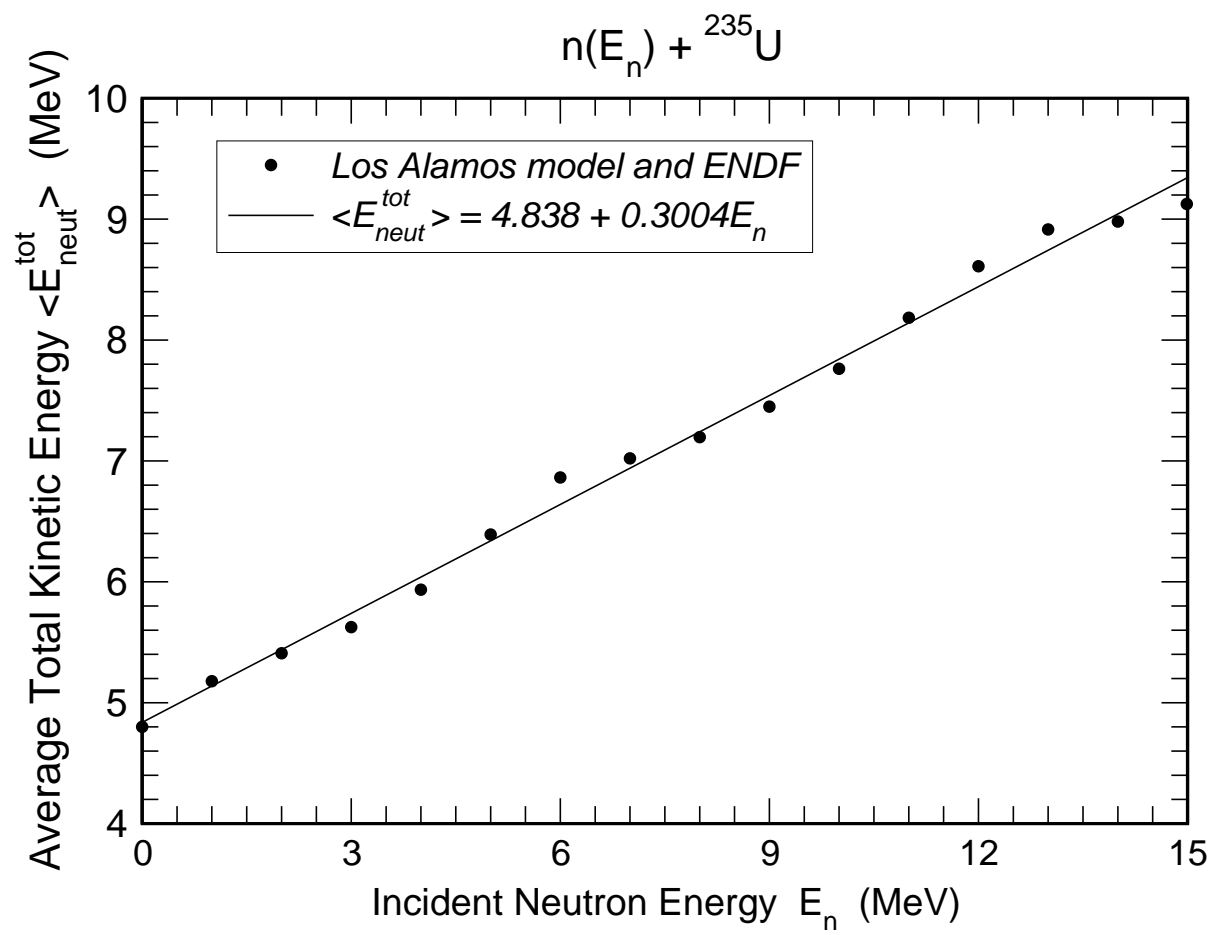

Fig. 20. Average total prompt fission neutron kinetic energy $\left\langle E_{\text {neut }}^{\text {tot }}\right\rangle$ for the $n\left(E_{n}\right)$ $+{ }^{235} \mathrm{U}$ system. 


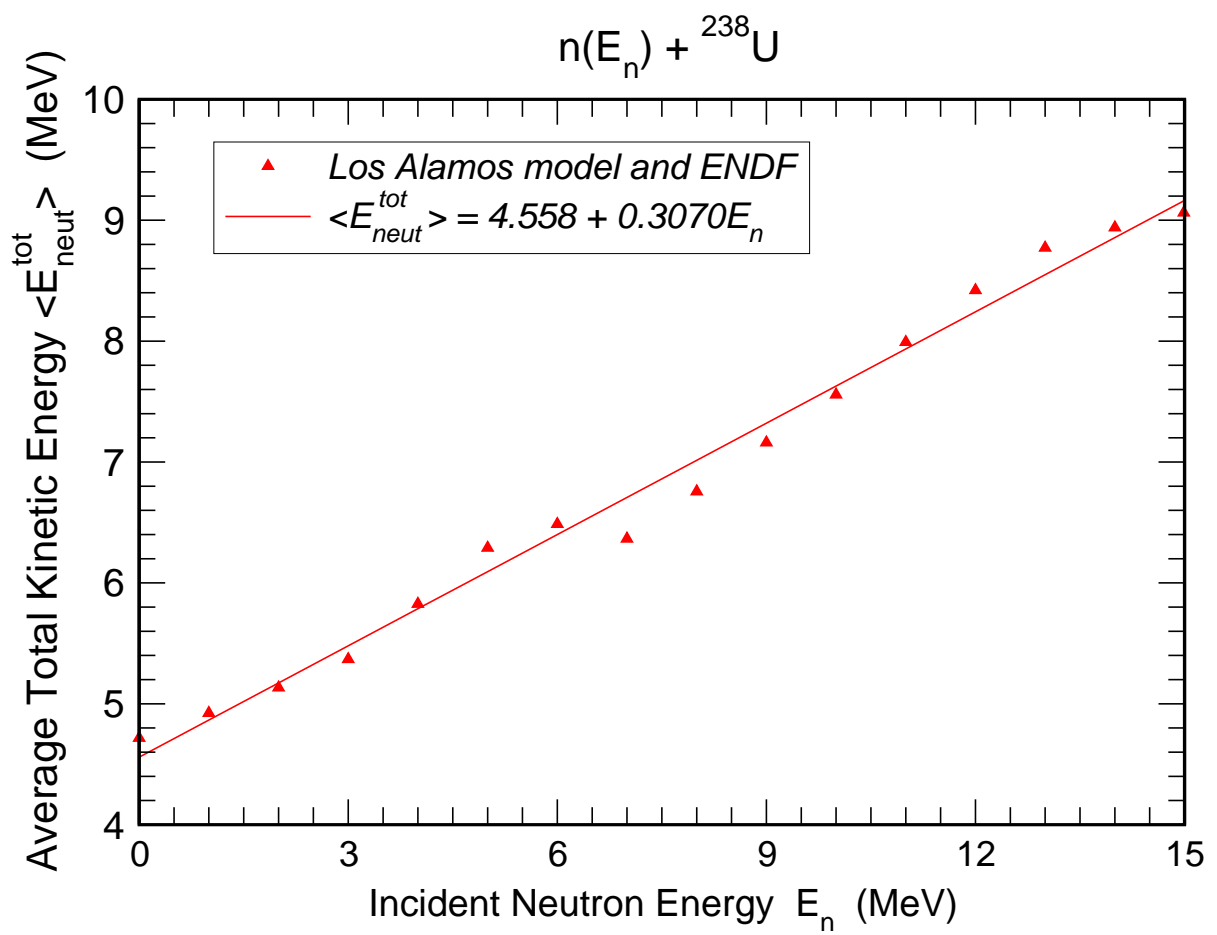

Fig. 21. Average total prompt fission neutron kinetic energy $\left\langle E_{\text {neut }}^{\text {tot }}\right\rangle$ for the $n\left(E_{n}\right)$ $+{ }^{238} \mathrm{U}$ system.

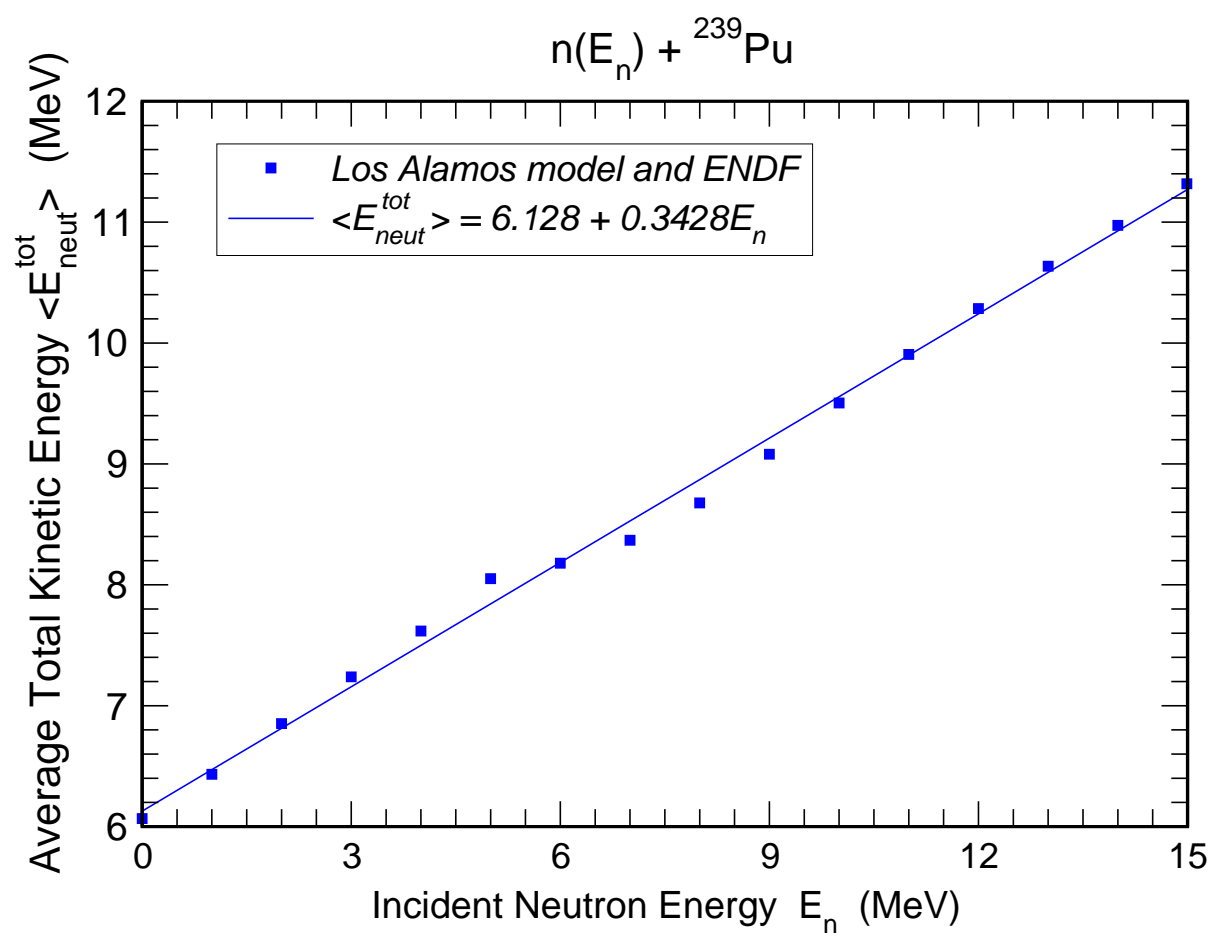

Fig. 22. Average total prompt fission neutron kinetic energy $\left\langle E_{\text {neut }}^{\text {tot }}\right\rangle$ for the $n\left(E_{n}\right)$ $+{ }^{239} \mathrm{Pu}$ system. 


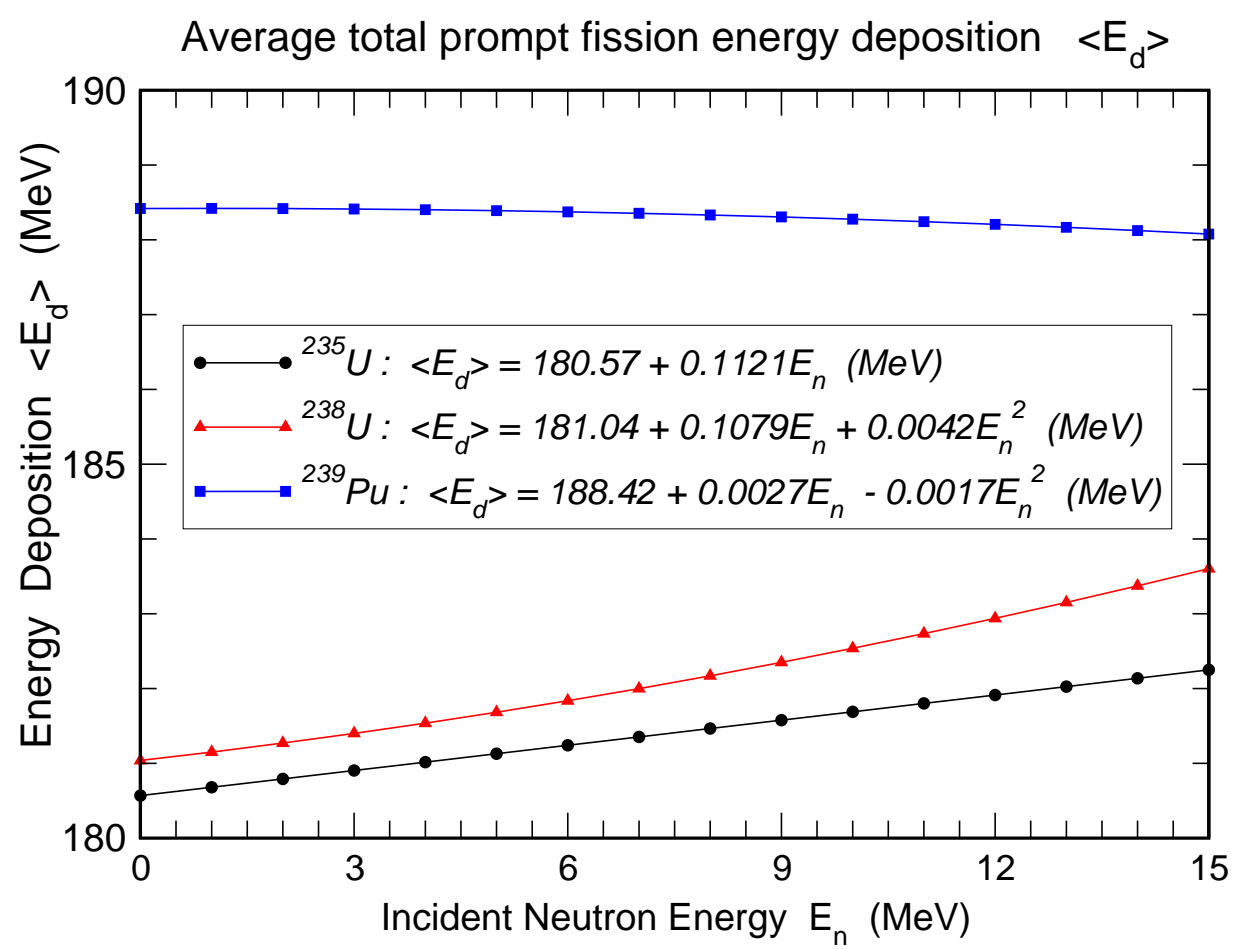

Fig. 23. Average total prompt fission energy deposition $\left\langle E_{d}\right\rangle$ for three systems.

Equation (37) for the average total prompt fission energy deposition $\left\langle E_{d}\right\rangle$ can now be evaluated for the three systems under study:

For the $\mathbf{n}+{ }^{235} \mathbf{U}$ system one substitutes Eq. (19), Eq. (43), and Eq. (28) into Eq. (37) to obtain

$$
\left\langle E_{d}\right\rangle=180.57+0.1121 E_{n}(\mathrm{MeV})
$$

For the $\mathbf{n}+{ }^{238} \mathbf{U}$ system one substitutes Eq. (21), Eq. (44), and Eq. (29) into Eq. (37) to obtain

$$
\left\langle E_{d}\right\rangle=181.04+0.1079 E_{n}+0.0042 E_{n}^{2}(\mathrm{MeV})
$$

For the $\mathbf{n}+{ }^{239} \mathbf{P u}$ system one substitutes Eq. (23), Eq. (45), and Eq. (30) into Eq. (37) to obtain

$$
\left\langle E_{d}\right\rangle=188.42+0.0027 E_{n}-0.0017 E_{n}^{2}(M e V) .
$$

These three equations for the average total prompt fission energy deposition are shown as a function of incident neutron energy in Fig. 23. The energy dependence of $\left\langle E_{d}\right\rangle$ is weak, increasing slightly with incident neutron energy for the two $U$ isotopes and decreasing very slightly for the $P u$ isotope. Again, 
the energy dependence is contrary to intuition and the explanation is the same as that for the energy release $\left\langle E_{r}\right\rangle$. However, the $\left\langle E_{d}\right\rangle$ energy dependence is stronger than that of $\left\langle E_{r}\right\rangle$ because the (positive) term $\left(E_{n}+B_{n}\right)$ wins over the (negative) term $\bar{\nu}_{p}\left\langle S_{n}\right\rangle$ in Eq. (34).

\section{Conclusions}

The total prompt fission energy release and energy deposition, together with their components, have been determined as a function of the kinetic energy of the neutron inducing the fission, for ${ }^{235} \mathrm{U},{ }^{238} \mathrm{U}$, and ${ }^{239} \mathrm{Pu}$. This study has relied primarily upon existing (published) experimental measurements and secondarily upon nuclear theory and nuclear models.

Contrary to basic physical intuition, it has been found that the energy release decreases somewhat with incident neutron energy and the energy deposition changes slightly with incident neutron energy. The main reason for this behavior is that symmetric fission increases with increasing incident neutron energy, but fission-fragment kinetic energies are at a minimum for symmetric fission. Even more striking is the fact that the $\mathrm{Q}$ values for fission are, on average, somewhat larger for symmetric fission than they are for asymmetric fission. The extra available fission-fragment excitation energy at symmetric fission, due to the smaller fragment kinetic energies and larger fission $\mathrm{Q}$ values, results in a peak in the $\bar{\nu}_{p}\left(A_{f}\right)$ vs. $A_{f}$ curve, that is, the prompt neutron multiplicities are peaked at symmetric fission.

These results would become more physically correct with a number of more complete and more accurate measurements over the incident neutron energy range. Then, instead of the simple linear and quadratic energy dependencies used here, more realistic characterizations of the incident neutron energy dependencies could be performed, for example, near the thresholds for multichance fission. In the $n+{ }^{238} U$ system this could already be done for the average total fission product kinetic energy $\left\langle T_{p}^{t o t}\right\rangle$, but is pointless to do so now because there are no measurements at all of the average total prompt fission gamma-ray energy $\left\langle E_{\gamma}^{\text {tot }}\right\rangle$ as a function of incident neutron energy for this same system. The current experimental data for fission product kinetic energies in the $n+{ }^{235} U$ and $n+{ }^{239} P u$ systems allow, at best, linear energy dependencies.

These results would also become more physically correct if the calculations performed using the Los Alamos model [5] were replaced by the identical calculations performed using a modern Hauser-Feshbach approach. Here the competition between neutron and gamma emission from fission fragments would be treated exactly and the angular momentum dependencies would be treated 
for each particle type emission. However, such an approach does not yet exist because not enough is known to adequately specify the fission fragment initial conditions across the fragment mass and charge distributions. Namely, (a) the partition of fissioning compound nucleus excitation energy between the light and heavy fragments, (b) the fragment initial angular momenta, and (c) the fragment initial parities, must all be specified for approximately 400 fragments. In addition, the fragment mass and charge yield distributions must be known as a function of incident neutron energy in order to properly weight the approximately 400 Hauser-Feshbach results for each incident neutron energy. There does not yet exist a calculation of these yield distributions of sufficient accuracy to perform this task, and measured fission product mass and charge yield distributions allowing construction of the corresponding fragment distributions are sufficiently complete at only two incident neutron energies: thermal and $14 \mathrm{MeV}$.

It is astonishing to find that after some 60 years of fission studies the postscission fission observables for the three major actinides ${ }^{235} \mathrm{U},{ }^{238} \mathrm{U}$, and ${ }^{239} \mathrm{Pu}$ have been so incompletely measured and understood. As a consequence of this work the following measurements are recommended for the three systems studied:

$$
\mathbf{n}+{ }^{235} \mathbf{U}
$$

(a) The average total fission-product kinetic energy should be measured from $10 \mathrm{keV}$ to $30 \mathrm{MeV}$. The existing data stops at about $9 \mathrm{MeV}$ and, furthermore, has uncertainties that need to be reduced.

(b) The average total prompt fission gamma-ray energy should be measured from $15 \mathrm{MeV}$ to $30 \mathrm{MeV}$ and several (three) of Frehaut's data points between 1 and $15 \mathrm{MeV}$ should be remeasured to verify the energy dependence.

(c) The prompt fission neutron spectrum (out to $15 \mathrm{MeV}$ emitted neutron energy) should be remeasured for incident neutron energies of $1,2,3$, and $5 \mathrm{MeV}$, and measured for $4 \mathrm{MeV}$ and the range $8 \mathrm{MeV}$ to $30 \mathrm{MeV}$.

$$
\mathbf{n}+{ }^{238} \mathbf{U}
$$

(a) The average total prompt fission gamma-ray energy should be measured from $10 \mathrm{keV}$ to $30 \mathrm{MeV}$. Apparently, no measurements exist.

(b) The prompt fission neutron spectrum (out to $15 \mathrm{MeV}$ emitted neutron energy) should be measured for incident neutron energies of 4, 10, and the range 12 to $30 \mathrm{MeV}$.

$$
\mathbf{n}+{ }^{239} \mathbf{P u}
$$

(a) The average total fission-product kinetic energy should be measured from about $3 \mathrm{MeV}$ to $30 \mathrm{MeV}$. The existing measurements become sparse at about $3.5 \mathrm{MeV}$ and stop just beyond $5 \mathrm{MeV}$. 
(b) The average total prompt fission gamma-ray energy should be measured from $10 \mathrm{keV}$ to $30 \mathrm{MeV}$. Only thermal measurements exist at the present time and the experimental content of Fort's evaluation is unknown.

(c) The prompt fission neutron spectrum (out to $15 \mathrm{MeV}$ emitted neutron energy) should be measured for incident neutron energies ranging from 4 $\mathrm{MeV}$ to $30 \mathrm{MeV}$.

It is manifestly clear that measured components of the above quantities for the light and heavy mass peaks as well as for near symmetric and far asymmetric fission will be doubly useful in serving both fundamental and applied postscission fission physics.

\section{Appendix A Multiple-Chance Fission Equations}

In the absence of the measured components of the average total prompt fission energy release $\left\langle E_{r}\right\rangle$ and average total prompt fission energy deposition $\left\langle E_{d}\right\rangle$ the following equations are used to calculate these quantities directly:

$$
\begin{aligned}
\left\langle E_{r}\right\rangle & =\frac{\left[P_{f_{1}}^{A}\left\langle E_{r}(A)\right\rangle+P_{f_{2}}^{A}\left\langle E_{r}(A-1)\right\rangle+P_{f_{3}}^{A}\left\langle E_{r}(A-2)\right\rangle\right]}{\left[P_{f_{1}}^{A}+P_{f_{2}}^{A}+P_{f_{3}}^{A}\right]} \\
\left\langle E_{d}\right\rangle & =\frac{\left[P_{f_{1}}^{A}\left\langle E_{d}(A)\right\rangle+P_{f_{2}}^{A}\left\langle E_{d}(A-1)\right\rangle+P_{f_{3}}^{A}\left\langle E_{d}(A-2)\right\rangle\right]}{\left[P_{f_{1}}^{A}+P_{f_{2}}^{A}+P_{f_{3}}^{A}\right]}
\end{aligned}
$$

where the total fission probability $P_{f}^{A}$ of the compound fissioning nucleus $A$ at excitation energy $\left[E_{n}+B_{n}(A)\right]$ is given by

$$
P_{f}^{A}\left[E_{n}+B_{n}(A)\right]=P_{f_{1}}^{A}\left[E_{n}+B_{n}(A)\right]+P_{f_{2}}^{A}\left[E_{n}+B_{n}(A)\right]+P_{f_{3}}^{A}\left[E_{n}+B_{n}(A)\right]
$$

in which $P_{f_{1}}^{A}$ is the probability for first-chance fission, the $(n, f)$ reaction, $P_{f_{2}}^{A}$ is the probability for second-chance fission, the $\left(n, n^{\prime} f\right)$ reaction, and $P_{f_{3}}^{A}$ is the probability for third-chance fission, the $\left(n, n^{\prime} n^{\prime \prime} f\right)$ reaction.

Correspondingly,

$$
\begin{gathered}
\left\langle E_{r}(A)\right\rangle=\left\langle T_{f}^{t o t}(A)\right\rangle+\bar{\nu}_{p}(A)\left[\left\langle S_{n}(A)\right\rangle+\left\langle\epsilon_{1}(A)\right\rangle\right]+\left\langle E_{\gamma}^{t o t}(A)\right\rangle-\left[E_{n}+B_{n}(A)\right] \\
\left\langle E_{d}(A)\right\rangle=\left\langle T_{p}^{t o t}(A)\right\rangle+\bar{\nu}_{p}(A)\langle E(A)\rangle+\left\langle E_{\gamma}^{\text {tot }}(A)\right\rangle
\end{gathered}
$$


which are evaluated as a function of excitation energy $\left[E_{n}+B_{n}(A)\right]$ of the $A$ system,

$$
\begin{aligned}
\left\langle E_{r}(A-1)\right\rangle & =\left\langle T_{f}^{t o t}(A-1)\right\rangle+\bar{\nu}_{p}(A-1)\left[\left\langle S_{n}(A-1)\right\rangle+\left\langle\epsilon_{2}(A-1)\right\rangle\right] \\
& +\left\langle E_{\gamma}^{t o t}(A-1)\right\rangle-\left[E_{n}-\left\langle\xi_{1}(A)\right\rangle\right]
\end{aligned}
$$

which are evaluated as a function of excitation energy $\left[E_{n}-\left\langle\xi_{1}(A)\right\rangle\right]$ of the $A-1$ system, and

$$
\begin{aligned}
\left\langle E_{r}(A-2)\right\rangle & =\left\langle T_{f}^{\text {tot }}(A-2)\right\rangle+\bar{\nu}_{p}(A-2)\left[\left\langle S_{n}(A-2)\right\rangle+\left\langle\epsilon_{3}(A-2)\right\rangle\right] \\
& +\left\langle E_{\gamma}^{t o t}(A-2)\right\rangle-\left[E_{n}-\left\langle\xi_{1}(A)\right\rangle-\left\langle\xi_{2}(A-1)\right\rangle\right. \\
& \left.-B_{n}(A-1)\right] \\
\left\langle E_{d}(A-2)\right\rangle= & \left\langle T_{p}^{t o t}(A-2)\right\rangle+\bar{\nu}_{p}(A-2)\langle E(A-2)\rangle+\left\langle E_{\gamma}^{t o t}(A-2)\right\rangle
\end{aligned}
$$

which are evaluated as a function of excitation energy $\left[E_{n}-\left\langle\xi_{1}(A)\right\rangle-\left\langle\xi_{2}(A-\right.\right.$ $\left.1)\rangle-B_{n}(A-1)\right]$ of the $A-2$ system.

All quantities appearing in the equations of this appendix are defined elsewhere in the text, mostly near Eq. (24) and Eqs. (41 - 42).

\section{References}

[1] I. Halpern, Annual Review of Nuclear Science 9 (1959) 245.

[2] F. Gönnenwein, in The Nuclear Fission Process, C. Wagemans Ed. (CRC Press, Boca Raton, FL, 1991) 287.

[3] L. Wilets, Theories of Nuclear Fission (Clarendon Press, Oxford, 1964) 21-22.

[4] Note that the upper limit, $\sim 10^{-7}$ [sec], may be application dependent and could, for example, be replaced by $\sim 10^{-8}$ or $\sim 10^{-6}$ [sec].

[5] D. G. Madland and J. R. Nix, Nucl. Sci. Eng. 81 (1982) 213. 
[6] Evaluated Nuclear Data File (ENDF), National Nuclear Data Center, Brookhaven National Laboratory, Upton, NY.

[7] C. Wagemans, in The Nuclear Fission Process, C. Wagemans Ed. (CRC Press, Boca Raton, FL, 1991) 545.

[8] J. W. Meadows and C. Budtz-Jorgensen, Argonne National Laboratory report ANL/NDM-64 (1982).

[9] Ch. Straede, C. Budtz-Jorgensen, and H. -H. Knitter, Nucl. Phys. A462 (1987) 85.

[10] R. Müller et al., Phys. Rev. C 29 (1984) 885.

[11] C. M. Zöller, Investigation of the neutron-induced fission of ${ }^{238} U$ in the energy range $1 \mathrm{MeV}$ to $500 \mathrm{MeV}$, Ph. D. thesis, Technische Hochschule Darmstadt, Darmstadt, Germany, 1995 (unpublished). Note that these experimental data are available from the Nuclear Data Section of the IAEA (Vienna).

[12] F. Vivès, F. -J. Hambsch, H. Bax, S. Oberstedt, Nucl. Phys. A662 (2000) 63.

[13] N. I. Akimov et al., Sov. J. Nucl. Phys. 13 (1971) 272.

[14] H. W. Schmitt, J. H. Neiler, and F. J. Walter, Phys. Rev. 141 (1966) 1146.

[15] J. H. Neiler, F. J. Walter, and H. W. Schmitt, Phys. Rev. 149 (1966) 894.

[16] J. Frehaut, A. Bertin, and R. Bois, Proc. Int. Conf. Nuclear Data for Science and Technology, Antwerp, Belgium, 6-10 September 1982, p. 78, D. Reidel Pub., Dordrecht, Holland (1983).

[17] D. C. Hoffman and M. M. Hoffman, Annual Review of Nuclear Science 24 (1974) 151.

[18] F. Pleasonton, Nucl. Phys. A213 (1973) 413.

[19] E. Fort, private communications (1994, 2004). 